Review

\title{
An Integrative Review of Recovery Services to Improve the Lives of Adults Living with Severe Mental Illness
}

\author{
Eric Badu ${ }^{1, *(\mathbb{D})}$, Anthony Paul O’Brien ${ }^{2}$ and Rebecca Mitchell ${ }^{3}$ \\ 1 School of Nursing and Midwifery, Faculty of Health and Medicine, The University of Newcastle, \\ Callaghan, NSW 2308, Australia \\ 2 Faculty of Health, Southern Cross University, East Lismore, NSW 2480, Australia; tony.obrien@scu.edu.au \\ 3 Faculty of Business and Economics, Macquarie University, Macquarie Park, NSW 2109, Australia; \\ rebecca.mitchell@mq.edu.au \\ * Correspondence: eric.badu@uon.edu.au; Tel.: +61-452414632
}

check for updates

Citation: Badu, E.; O’Brien, A.P.; Mitchell, R. An Integrative Review of Recovery Services to Improve the Lives of Adults Living with Severe Mental Illness. Int. J. Environ. Res. Public Health 2021, 18, 8873. https:// doi.org/10.3390/ijerph18168873

Academic Editor: Lonnie R. Snowden

Received: 1 June 2021

Accepted: 18 August 2021

Published: 23 August 2021

Publisher's Note: MDPI stays neutral with regard to jurisdictional claims in published maps and institutional affiliations.

Copyright: (c) 2021 by the authors. Licensee MDPI, Basel, Switzerland. This article is an open access article distributed under the terms and conditions of the Creative Commons Attribution (CC BY) license (https:/ / creativecommons.org/licenses/by/ $4.0 /)$.

\begin{abstract}
There is an increasing call for recovery-oriented services but few reviews have been undertaken regarding such interventions. This review aims to synthesize evidence on recovery services to improve the lives of adults living with severe mental illness. An integrative review methodology was used. We searched published literature from seven databases: Medline, EMBASE, PsycINFO, CINAHL, Google Scholar, Web of Science, and Scopus. Mixed-methods synthesis was used to analyse the data. Out of 40 included papers, $62.5 \%$ (25/40) used quantitative data, $32.5 \%$ used qualitative and 5\% (2/40) used mixed methods. The participants in the included papers were mostly adults with schizophrenia and schizoaffective disorder. This review identified three recoveryoriented services-integrated recovery services, individual placement services and recovery narrative photovoice and art making. The recovery-oriented services are effective in areas such as medication and treatment adherence, improving functionality, symptoms reduction, physical health and social behaviour, self-efficacy, economic empowerment, social inclusion and household integration. We conclude that mental health professionals are encouraged to implement the identified recovery services to improve the recovery goals of consumers.
\end{abstract}

Keywords: recovery; rehabilitation; systematic reviews; mental health; nursing

\section{Introduction}

Recovery in mental health is defined as the process of restoration of functioning and well-being, improvements in quality of life of an individual diagnosed with longerterm mental health problems or emotional difficulties, using a whole-system or integrated approach [1-4]. The concept of recovery has been defined according to two main categories-clinical recovery (e.g., service-based, or objective definition) and personal recovery (subjective) [1-4]. The two forms of recovery-clinical and personal-are relevant in physical and mental conditions. The personal perspective considers recovery as a journey or process, whilst the clinical perspectives consider it as an outcome $[2,5]$. Personal recovery is a continuous process involving personal growth and development, improvement of symptoms, regaining control, and establishing a personally fulfilling and meaningful life, and incorporates restoration to a healthy state $[1,3,5]$. The concept also encapsulates a growing sense of agency and autonomy [5]. Some studies have defined personal recovery as pursuing one's personal hopes, aspirations, personal abilities, and in the achievement of a personally acceptable quality of life [1,6,7]. Furthermore, some evidence has suggested that personal recovery should be given attention to provide holistic mental health services [4,8]. In particular, the individual's values and preferences for specific treatments or other forms of support should be central [4]. Conversely, clinical recovery, which is based on a medical perspective, includes remission of symptoms or a return to normal functioning and is less holistic $[1,3,9]$. 
A broad range of recovery services are being promoted worldwide in hospitals and community settings. Such services are operationalized as interventions that aim to provide person-centred mental health services, thus a multidimensional process of transformation involving positive transitions. The primary aim of recovery services is to help individuals to recover and develop some social and intellectual skills needed to live, learn and work in the community [2,5-7]. Recovery services help to improve psychological, social and cognitive functioning, social inclusion, and positive thinking of people with mental illness $[1-4,10,11]$. Despite this, past evidence has shown that several multi-level systemic and individual consumer factors continue to impede recovery services [2-5]. The systemic factors are mostly associated with the strengths of psychiatric facilities (patient loads, time constraints in clinical encounters), the capacity of mental health professionals (ancillary professionals and training support), governance or leadership of mental health services (management resistance to change and comprehensive multidisciplinary and inter-service collaboration), affordability, and inclusivity of the mental health services [2-5,11]. Additionally, individual factors include the ability to ensure early access to services, and normative life pursuits such as education, employment, sexuality, friendship, spirituality, and religious participation [2,5].

Several empirical studies regarding recovery services are being conducted recently to promote the personal recovery journey of consumers. The evidence has largely addressed services used to foster integrated recovery services. Only a few of such studies have attempted to systematically review the literature regarding recovery services for adults with severe mental illness (SMI). A preliminary search we conducted showed that though some existing reviews have been conducted regarding recovery, they are limited to the general mental health population and some specific services, such as individual placement services $[10,12,13]$. Critically, no review study has been undertaken to aggregate a synthesis of both qualitative and quantitative studies regarding recovery services that promote the personal recovery journey of adults living with severe mental illness. Adults with serious mental illness (SMI) may suffer from a long-term condition that requires a special integrated or whole-system approach to treatment. This review, therefore, aimed to: (1) identify existing evidence on recovery services; and to (2) synthesize evidence on the outcome of recovery services for adults living with severe mental illness.

The review findings are significant for several reasons. The evidence is expected to inform policy decision making on the well-being of adults with serious mental illness. The evidence is also considered to be valuable to policy makers and mental health professionals to strengthen mental health services. Finally, the evidence can guide researchers and clinicians in terms of future research and further inform the training of mental health professionals and students.

\section{Methods}

\subsection{Methodology}

An integrative review is an approach that allows simultaneous inclusion of diverse methodologies (e.g., qualitative and quantitative data) and varied perspectives to fully understand the phenomenon of concern [14,15]. This review study aims to use diverse data sources to develop a holistic understanding of recovery services for adults with severe mental illness. This review method contributes greatly to evidence-based practice for mental health nursing. This review employed a five-stage process-problem identification (developing and defining research question and study aim); searching literature (incorporating a comprehensive search strategy); evaluation of data (assessing for methodological quality); analysis of extracted data (data reduction, display, comparison and conclusions) and presentation (mixed-methods synthesis implications for practice, policy and research) [14].

\subsection{Inclusion Criteria}

This review included studies that address recovery services for adults (e.g., 18 years and above) living with severe mental illness (e.g., in-patients, out-patients, community-based residential services, home-based services). This review defined individuals with serious mental 
illness as those with a mental, behavioural, or emotional disorder resulting in serious functional impairment, which substantially interferes with or limits one or more major life activities [16]. Adults with serious mental illness were individuals with schizophrenia, bipolar disorder, mania, or psychosis that have been diagnosed by a health professional and self-reported or by proxy [17]. Studies that were included targeted services such as disability support and recovery services. This study also included papers that address the effect of recovery services on the lives of people living with severe mental illness.

This review included papers of all methods and designs. Papers included used mixed methods as well as quantitative and qualitative methods. The quantitative methods included quantitative randomized controlled trials, quantitative non-randomized designs (analytical cross-sectional) and quantitative descriptive studies. Additionally, the qualitative papers used ethnography and participatory methodology, grounded theory, phenomenology, and narrative. This review only considered studies published in the English language from January 2008 to January 2020 - a period of increased research into recovery services and interventions for adults with mental illness.

\subsection{Exclusion Criteria}

Papers that were excluded are based purely on general health services or clinical effectiveness of a particular intervention with no connection to recovery services and mental health rehabilitation. Additionally, papers were excluded if they address recovery services for children and adolescents, workplace mental health issues, recovery services in stroke patients or traumatic injury. Other general exclusion criteria were systematic reviews, conference abstracts, clinical case reviews, book chapters, papers that present opinions, editorials and commentaries.

\subsection{Search Strategy and Selection Procedure}

We searched seven electronic databases-EMBASE, CINAHL (EBSCO), Web of Science, Scopus, PsycINFO, Medline and Google Scholar. The search was conducted according to the Joanna Briggs Institute (JBI) recommended guidelines for conducting systematic reviews [18]. A three-stage search strategy was utilised to search for information (see Table 1). An initial limited search was conducted in EMBASE and MEDLINE (Table 1). The initial search was not restricted by limiters-field, language, timespan and type of publication. We analysed the text words contained in the title and abstract and the index terms from the initial search results [18]. A second search using all identified keywords and index terms was then conducted across all remaining databases. These searches were restricted to title, abstract and keywords due to a plethora of references obtained through the initial search. Finally, the reference lists of all identified articles were hand searched for additional studies [18].

The selection of eligible articles adhered to the Preferred Reporting Items for Systematic Reviews and Meta-Analyses (PRISMA) [19] (Figure 1). Firstly, three authors independently screened the titles of articles that were retrieved and then approved those meeting the selection criteria. The authors reviewed all titles and abstracts and agreed on those needing full-text screening. The first author conducted the initial screening of titles and abstracts. The second and third authors conducted the second screening of titles and abstracts of all the identified papers. The authors conducted full-text screening according to the inclusion and exclusion criteria. 
Table 1. Search strategy and selection procedure.

Stages

Stage 1 (initial search in MEDLINE and EMBASE)

Stage 2 (search across CINAHL, Web of Science, Scopus, and PsycINFO)
Search Terms and Keywords

(Disabilit* or "psychosocial disability" or Adult or "mental disorders" or

"mental illness" or "mental condition").mp. AND ("Social Support" or

"Individual Support" or "disability support" or "social inclusion" or integration or "community acceptance" or participation).mp. AND ("nursing homes" or "residential facilities" or "residential care" or "rehabilitation centers" or "community rehabilitation" or "residential program" or "residential care" or institutions or residential treatment).mp. AND ("service model" or "service typology" or rehabilitation or rehabilitat * or "disabled persons" or vocational or "psychosocial support" or or "psychosocial deprivation").mp. AND (recovery or effectiv * or "patient reported" or "outcome measures" or

"treatment outcome" or "patient outcome" or assessment or functioning or "quality of life" or coping or "patient-centered care").mp

(Disabilit * or "psychosocial disability" or Adult or "ental disorders" or

"mental illness" or "mental condition").mp. AND ("social support" or

"individual support" or "disability support" or "social inclusion" or integration or "community acceptance" or participation).mp. AND ("nursing homes" or "residential facilities" or "residential care" or "rehabilitation centers" or "community rehabilitation" or "residential program" or "residential care" or institutions or residential treatment).mp. AND ("service model" or "service typology" or rehabilitation or rehabilitat * or "disabled persons" or vocational or "psychosocial support" or or "psychosocial deprivation").mp. AND (recovery or effectiv * or "patient reported" or "outcome measures" or

"treatment outcome" or "patient outcome" or assessment or functioning or "quality of life" or coping or "patient-centered care").mp

\subsection{Data Management and Extraction}

Endnote X8 (software) was used to manage the search results, screening, reviewing articles, as well as removing duplicate references. Three reviewers independently managed the data extraction process [18]. The authors developed a data extraction form to handle all aspects of data extraction (Table S1). The data extraction form was developed according to the Cochrane and the Joanna Briggs Institute (JBI) manuals [18] for systematic reviews as well as consultation with experts in methodologies and the subject area [18]. The authors extracted results of the included papers in numerical, tabular and textual format [18]. The first author conducted the data extraction whilst the second and third authors conducted the second review of the extracted data. The data extraction focused on study details (citation, year of publication, author, contact details of lead author, and funder/sponsoring organization), publication source, methodological characteristics, study population, subject area (e.g., recovery service model, recovery concept, recovery intervention, period of project implementation, phases or components of recovery intervention, outcome or impact of intervention), as well as additional information and recommendations and other potential references to follow up.

\subsection{Assessment of Methodological Quality}

The authors developed a critical appraisal checklist using the Mixed-Methods Appraisal Tool (MMAT) [20] and the Joanna Briggs Institute (JBI) [21] critical appraisal tool. The critical appraisal checklist was used by the authors (the first, second and third authors) to assess the methodological quality of the included papers. The critical appraisal tool was sub-divided into sections such as study details, methodology (e.g., categorized as qualitative, quantitative randomized controlled trials, quantitative non-randomized, including cohort study, case-control study, analytical cross-sectional, quantitative descriptive, and 
mixed methods) and overall quality score (Table S2). The methodological quality score was rated as low quality if the overall score was below $25 \%$, medium quality if $50 \%$ and high quality $70 \%$ and above. The scores were computed by summing the number of 'Yes' counts in each sub-section of the methodological criteria. The total score was then expressed as a percentage [20].

\section{Data Synthesis}

The extracted data were analysed using mixed-methods synthesis [14,18]. Mixedmethods synthesis seeks to develop an aggregated synthesis of qualitative and quantitative data [18]. The process involves familiarization with the data, generating initial codes, searching for themes, reviewing themes, defining and naming themes and producing a thematic chart $[22,23]$. The authors coded the quantitative and qualitative data together. Data display matrices were developed to document all of the coded data from each extracted data [14]. Alphabets and colours were assigned to each of the coded ideas. The resulting codes from quantitative and qualitative data were used to generate descriptive themes [18] The descriptive themes were categorized into global and organizing themes. The themes have been discussed with the concepts and theoretical constructs that explain recovery services in mental health. The background information of included papers was analysed using STATA version 15.

\section{Results}

Description of Retrieved Papers

This review retrieved 788 papers from all databases. Of these, 94 duplicates were removed. The titles and abstracts of 694 non-duplicate articles were screened for eligibility, after which 266 were excluded. A total of 428 full-text articles were assessed for eligibility (376 were excluded). This review extracted data from 52 full-text articles that met the eligibility. Of these, 2 articles were identified through hand searching the reference list. Overall, 40 papers were included in the final synthesis (Figure 1). Out of the 40 papers, 38 met the criteria for high methodological quality assessment, whilst only two papers had medium quality. 


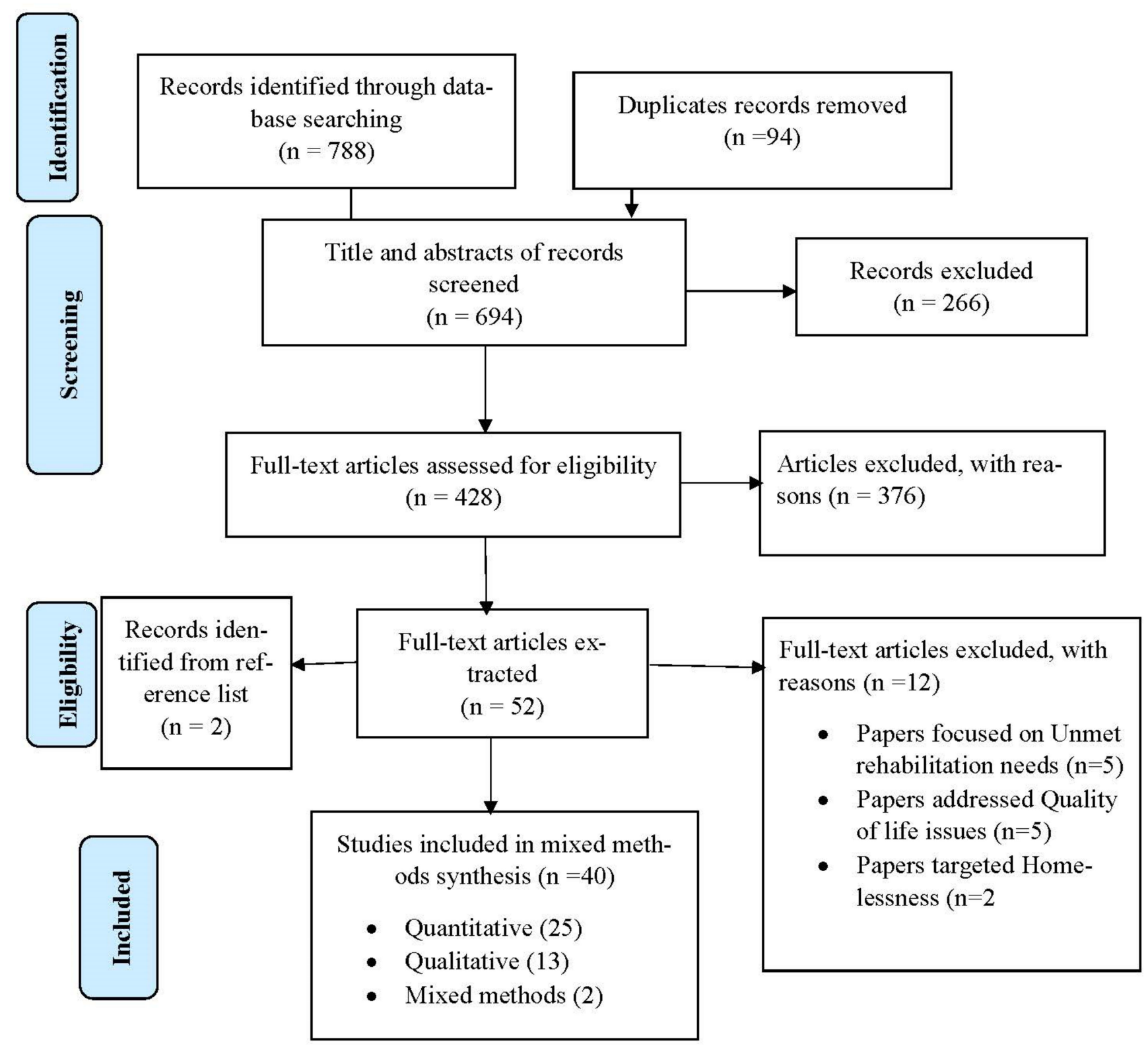

Figure 1. Flow chart of included papers.

\section{Characteristics of Included Articles}

More than half of the papers $(23 / 40 ; 57.5 \%)$ were interventional studies. Of these, more than one-third $(12 / 29 ; 43.47 \%)$ used randomized controlled trials. More than half of the included papers $(25 / 40 ; 62.5 \%)$ used quantitative methods, $32.5 \%$ used qualitative methods and $5 \%(2 / 40)$ used mixed methods. The participants in the included papers were mostly adults with schizophrenia and schizoaffective disorder (Table 2). Approximately $57.5 \%(23 / 40)$ of the included papers used descriptive and inferential statistics, $17.5 \%(7 / 40)$ used thematic analysis, and 5\% (2/40) each used descriptive statistics alone, grounded theory and triangulation (thematic, descriptive and inferential statistics). In addition, 27.5\% $(11 / 40)$ of the included papers were studies conducted in the USA, $10 \%(4 / 40)$ were studies conducted across six European centres (UK, The Netherlands, Germany, Italy, Bulgaria and Switzerland), 10\% (4/40) were conducted in Sweden, 7.5\% (3/40) targeted Hong Kong and $5 \%(2 / 40)$ each focused on Canada, China, South Africa and the Netherlands (see Table 2). 
Table 2. Characteristics of included papers.

\begin{tabular}{|c|c|c|c|c|c|c|c|c|c|}
\hline Papers & Objectives & Setting & Age/Gender & Participants & $\begin{array}{c}\text { Study De- } \\
\text { sign/Methods }\end{array}$ & Data Collection Instrument & Analysis & $\mathrm{Q}^{+}$ & Summary of Article \\
\hline $\begin{array}{c}\text { Asher, Hanlon } \\
\text { [24] }\end{array}$ & $\begin{array}{l}\text { The acceptability and } \\
\text { feasibility of CBR in } \\
\text { practice as well as how } \\
\text { CBR may improve } \\
\text { functioning among } \\
\text { people with } \\
\text { schizophrenia }\end{array}$ & Ethiopia & $\begin{array}{c}\text { Mean age }=39.5 \\
\text { males and } \\
\text { females }\end{array}$ & Schizophrenia & $\begin{array}{c}\text { Quasi- } \\
\text { experimental } \\
\text { design/Mixed } \\
\text { methods }\end{array}$ & $\begin{array}{ll}\text { - } & \text { In-depth interviews (IDIs) } \\
& \text { Discrimination and Stigma } \\
& \text { Scale-12 (DISC-12) } \\
\text { - } & \text { Alcohol Use Disorders } \\
& \text { Identification Test (AUDIT) } \\
\text { - } & \text { Patient Health } \\
& \text { Questionnaire-9(PHQ-9) } \\
\text { - } & \text { Involvement Evaluation } \\
& \text { Questionnaire (IEQ) } \\
\text { - } & \text { World Health Organization } \\
& \text { Disability Assessment } \\
& \text { Schedule (WHODAS) } 2.0 \\
\text { - } & \text { Clinical Global Impression }\end{array}$ & $\begin{array}{c}\text { Thematic } \\
\text { analysis and } \\
\text { descriptive } \\
\text { statistics }\end{array}$ & $\mathrm{H}$ & $\begin{array}{l}\text { CBR program has the } \\
\text { capacity to improve } \\
\text { functioning of people } \\
\text { with schizophrenia }\end{array}$ \\
\hline $\begin{array}{l}\text { Brooke-Sumner, } \\
\text { Lund [25] }\end{array}$ & $\begin{array}{c}\text { To develop a } \\
\text { community-based } \\
\text { psychosocial } \\
\text { rehabilitation program } \\
\text { for service users with } \\
\text { schizophrenia }\end{array}$ & South Africa & $\begin{array}{l}\text { Maximum age }= \\
45 ; \text { females }\end{array}$ & Schizophrenia & $\begin{array}{c}\text { Quasi- } \\
\text { experimental } \\
\text { de- } \\
\text { sign/Qualitative }\end{array}$ & - In-depth interviews (IDIs) & $\begin{array}{l}\text { Thematic } \\
\text { analysis }\end{array}$ & $\mathrm{H}$ & $\begin{array}{l}\text { The program } \\
\text { improved the lives of } \\
\text { service users with } \\
\text { schizophrenia—self- } \\
\text { esteem, social support, } \\
\text { illness knowledge, } \\
\text { self-care, and } \\
\text { contribution to their } \\
\text { households }\end{array}$ \\
\hline $\begin{array}{l}\text { Brooke-Sumner, } \\
\text { Selohilwe [26] }\end{array}$ & $\begin{array}{l}\text { Investigated a non- } \\
\text { specialist-delivered } \\
\text { program for } \\
\text { psychosocial } \\
\text { rehabilitation for } \\
\text { service users with } \\
\text { schizophrenia in a } \\
\text { low-resource South } \\
\text { African setting }\end{array}$ & South Africa & $\begin{array}{l}\text { Age range }= \\
21-44 ; \text { males } \\
\text { and females }\end{array}$ & Schizophrenia & $\begin{array}{c}\text { Quasi- } \\
\text { experimental } \\
\text { design/Mixed } \\
\text { methods }\end{array}$ & $\begin{array}{ll}- & \text { In-depth interviews (IDIs) } \\
- & \text { Brief Psychiatric Rating Scale } \\
& \text { (BPRS) } \\
\text { - } & \text { Clinician-administered scale } \\
- & \text { World Health Organization } \\
& \text { Disability Assessment Scale } \\
& \text { (WHODAS) } \\
\text { - } & \text { Stigma of Mental Illness } \\
& \text { Inventory (ISMI) }\end{array}$ & $\begin{array}{c}\text { Thematic } \\
\text { analysis and } \\
\text { inferential } \\
\text { statistics }\end{array}$ & $\mathrm{H}$ & $\begin{array}{l}\text { The program achieved } \\
\text { reduction in ISMI } \\
\text { assessment as well as } \\
\text { improved } \\
\text { psychosocial } \\
\text { well-being of service } \\
\text { users with } \\
\text { schizophrenia }\end{array}$ \\
\hline
\end{tabular}


Table 2. Cont.

\begin{tabular}{|c|c|c|c|c|c|c|c|c|c|}
\hline Papers & Objectives & Setting & Age/Gender & Participants & $\begin{array}{c}\text { Study De- } \\
\text { sign/Methods }\end{array}$ & Data Collection Instrument & Analysis & $\mathrm{Q}^{+}$ & Summary of Article \\
\hline $\begin{array}{l}\text { Browne and } \\
\text { Waghorn [27] }\end{array}$ & $\begin{array}{l}\text { to retrospectively } \\
\text { assess the } \\
\text { implementation of IPS } \\
\text { practices and youth } \\
\text { employment outcomes }\end{array}$ & New Zealand & $\begin{array}{l}\text { Age range }= \\
16-25 ; \text { males } \\
\text { and females }\end{array}$ & $\begin{array}{c}\text { Affective } \\
\text { (including } \\
\text { comorbid } \\
\text { anxiety), bipolar } \\
\text { affective } \\
\text { disorder, } \\
\text { generalized } \\
\text { anxiety disorder }\end{array}$ & $\begin{array}{c}\text { Observational } \\
\text { design } \\
\text { (retrospective } \\
\text { case study)/ } \\
\text { Quantitative }\end{array}$ & - Case review & $\begin{array}{l}\text { Descriptive } \\
\text { statistics }\end{array}$ & M & $\begin{array}{l}\text { The IPS program was } \\
\text { effective in terms of } \\
\text { the proportion of } \\
\text { young clients } \\
\text { commencing } \\
\text { competitive } \\
\text { employment, and } \\
\text { duration of longest job } \\
\text { held }\end{array}$ \\
\hline $\begin{array}{c}\text { Burns, White } \\
\text { [28] }\end{array}$ & $\begin{array}{c}\text { The acceptability and } \\
\text { effectiveness of IPS in } \\
\text { Europe }\end{array}$ & $\begin{array}{l}\text { Five European } \\
\text { countries }\end{array}$ & Mean age $=38$ & $\begin{array}{c}\text { schizophrenia or } \\
\text { schizoaffective } \\
\text { disorder bipolar } \\
\text { disorder }\end{array}$ & $\begin{array}{l}\text { Randomized } \\
\text { controlled } \\
\text { trial/Quantitative }\end{array}$ & - Questionnaire & $\begin{array}{l}\text { Inferential } \\
\text { statistics }\end{array}$ & High & $\begin{array}{c}\text { Individual Placement } \\
\text { and Support (IPS) was } \\
\text { approximately } \\
\text { two-fold more } \\
\text { effective than } \\
\text { vocational services in } \\
\text { returning to open } \\
\text { employment }\end{array}$ \\
\hline $\begin{array}{c}\text { Catty, Lissouba } \\
\text { [29] }\end{array}$ & $\begin{array}{l}\text { Determine which } \\
\text { patients with severe } \\
\text { mental illness do well } \\
\text { in vocational services } \\
\text { and which process and } \\
\text { service factors are } \\
\text { associated with better } \\
\text { outcomes }\end{array}$ & $\begin{array}{l}\text { Six European } \\
\text { centres }\end{array}$ & $\begin{array}{l}\text { Age range }= \\
\text { 18-local } \\
\text { retirement age; } \\
\text { males and } \\
\text { females }\end{array}$ & Schizophrenia & $\begin{array}{l}\text { Randomized } \\
\text { controlled } \\
\text { trial/Quantitative }\end{array}$ & $\begin{array}{ll}\text { - } & \text { Global Assessment of } \\
& \text { Functioning-Symptoms } \\
\text { (GAF-S) and Disability } \\
\text { (GAF-D) } \\
\text { - } \quad \text { Positive and Negative } \\
\text { Syndrome Scale } \\
\text { - } \quad \text { Hospital Anxiety and } \\
\text { Depression Scale } \\
\text { - } \quad \text { Groningen Social Disability } \\
\text { - Schedule } \\
\text { Lancashire Quality of Life } \\
\text { - Profile-European Version } \\
\text { - } \quad \text { Rosenberg Self-Esteem Scale } \\
\text { Camberwell Assessment of } \\
\text { Need-European short } \\
\text { version }\end{array}$ & $\begin{array}{l}\text { Inferential } \\
\text { statistics }\end{array}$ & $\mathrm{H}$ & $\begin{array}{c}\text { The IPS services were } \\
\text { more effective than the } \\
\text { vocational services for } \\
\text { every vocational } \\
\text { outcome }\end{array}$ \\
\hline
\end{tabular}


Table 2. Cont.

\begin{tabular}{|c|c|c|c|c|c|c|c|c|c|}
\hline Papers & Objectives & Setting & Age/Gender & Participants & $\begin{array}{c}\text { Study De- } \\
\text { sign/Methods }\end{array}$ & Data Collection Instrument & Analysis & $\mathrm{Q}^{+}$ & Summary of Article \\
\hline $\begin{array}{c}\text { Chang, Chen } \\
\text { [30] }\end{array}$ & $\begin{array}{l}\text { Investigated the effect } \\
\text { of a music-creation } \\
\text { group program on the } \\
\text { anxiety, self-esteem, } \\
\text { and quality of life of } \\
\text { patients with SMI }\end{array}$ & Taiwan & $\begin{array}{l}\text { Age range } \\
20-65 ; \text { males } \\
\text { and females }\end{array}$ & $\begin{array}{l}\text { Schizophrenia } \\
\text { or affective } \\
\text { disorders }\end{array}$ & $\begin{array}{c}\text { Quasi- } \\
\text { experimental } \\
\text { design/ } \\
\text { Quantitative }\end{array}$ & $\begin{array}{ll}- & \text { Demographic data } \\
- & \text { Hamilton Anxiety Rating } \\
& \text { Scale (HAM-A) } \\
\text { - } & \text { Rosenberg Self-Esteem Scale } \\
& \text { (RSES) } \\
\text { - } & \text { World Health Organization } \\
& \text { Quality of Life-BREF } \\
\text { (WHOQOL-BREF) }\end{array}$ & $\begin{array}{l}\text { Inferential } \\
\text { statistics }\end{array}$ & $\mathrm{H}$ & $\begin{array}{l}\text { Participating in a } \\
\text { structured } \\
\text { music-creation } \\
\text { intervention improved } \\
\text { the psychological } \\
\text { well-being, } \\
\text { self-esteem, quality of } \\
\text { life and social } \\
\text { relationship of } \\
\text { consumers with SMI }\end{array}$ \\
\hline Chiu, Ho [31] & $\begin{array}{l}\text { To test empirically the } \\
\text { Substance Abuse and } \\
\text { Mental Health } \\
\text { Services } \\
\text { Administration } \\
\text { (SAMHSA) recovery } \\
\text { model }\end{array}$ & Hong Kong & $\begin{array}{c}\text { Mean age }=41.6 \\
\text { males and } \\
\text { females }\end{array}$ & $\begin{array}{l}\text { Schizophrenia } \\
\text { spectrum } \\
\text { disorder }\end{array}$ & $\begin{array}{l}\text { Cross-sectional/ } \\
\text { Quantitative }\end{array}$ & $\begin{array}{ll}\text { - } & \text { Internalized Stigma of } \\
\text { - } & \text { Mental Illness scale (ISMI) } \\
\text { - } & \text { Mesilience Scale (RS) } \\
& \text { Emping Decision } \\
\text { - } & \text { Exercise of Self-Care Agency } \\
& \text { Scale } \\
\text { - } & \text { Mastery Scale (MS) } \\
\text { - } & \text { Adult State Hope Scale } \\
& \text { (ASHS) } \\
\text { - } & \text { Health Care Climate } \\
\text { - } & \text { Recovionnaire (HCCQ) } \\
\text { - } & \text { Questionnaire (RAQ-7) } \\
\text { Medical Outcome Study } \\
\text { Social Support } \\
\text { Survey-Chinese version } \\
\text { (EISS-MOS-SSS-C) } \\
\text { Schizophrenia Quality of Life } \\
\text { Scale (SQLS) } \\
\text { Multidimensional Scale of } \\
\text { Perceived Social } \\
\text { Support-Chinese version } \\
\text { (MSPSS-C) } \\
\text { World Health Organization } \\
\text { Spirituality Religion and } \\
\text { Personal Belief Scale-Hong } \\
\text { Kong version } \\
\text { (WHO-SRPBHK) }\end{array}$ & $\begin{array}{l}\text { Inferential } \\
\text { statistics }\end{array}$ & $\mathrm{H}$ & $\begin{array}{l}\text { Psychosocial } \\
\text { symptoms, respect, } \\
\text { resilience, and } \\
\text { empowerment were } \\
\text { significant } \\
\text { contributors of } \\
\text { recovery }\end{array}$ \\
\hline
\end{tabular}


Table 2. Cont.

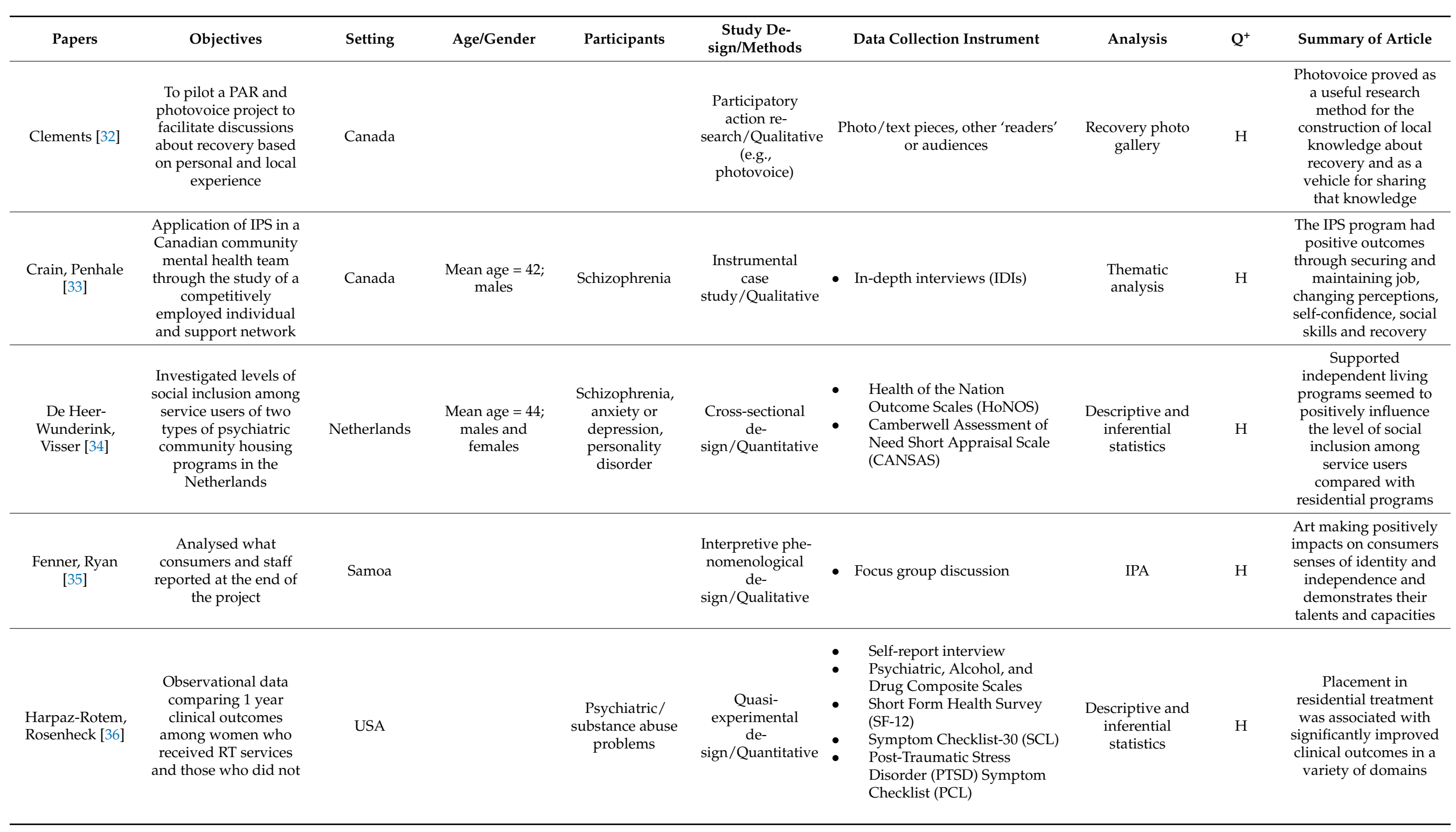


Table 2. Cont.

\begin{tabular}{|c|c|c|c|c|c|c|c|c|c|}
\hline Papers & Objectives & Setting & Age/Gender & Participants & $\begin{array}{l}\text { Study De- } \\
\text { sign/Methods }\end{array}$ & Data Collection Instrument & Analysis & $\mathrm{Q}^{+}$ & Summary of Article \\
\hline $\begin{array}{c}\text { Hultqvist, } \\
\text { Markstrom [37] }\end{array}$ & $\begin{array}{l}\text { Comparing users of } \\
\text { two approaches to } \\
\text { psychosocial } \\
\text { rehabilitation in } \\
\text { Sweden, } \\
\text { community-based } \\
\text { mental health day } \\
\text { centres (DCs) and } \\
\text { clubhouses }\end{array}$ & Sweden & $\begin{array}{c}\text { Mean age }=48.7 \\
\text { males and } \\
\text { females }\end{array}$ & $\begin{array}{l}\text { Psychoses, } \\
\text { mood and } \\
\text { anxiety } \\
\text { disorders, } \\
\text { autism// } \\
\text { neuropsychiatric } \\
\text { disorders }\end{array}$ & $\begin{array}{c}\text { Quasi- } \\
\text { experimental } \\
\text { de- } \\
\text { sign/Quantitative }\end{array}$ & $\begin{array}{ll}\text { - } & \text { Manchester Short } \\
\text { Assessment of Quality of Life } \\
\text { (MANSA) } \\
\text { - } \quad \text { Self-Esteem Rosenberg } \\
\text { - } & \text { Global Assessment of } \\
& \text { Functioning (GAF) } \\
\text { - } & \text { MOS 36-item Short-Form } \\
& \text { Health Survey } \\
\text { - } & \text { Social Interaction (ISSI) } \\
- & \text { Satisfaction with Daily } \\
\text { - } & \text { Sccupations (SDO) } \\
-\quad & \text { Thedish version of the CSQ-8 } \\
\text { - } & \text { Socio-demographic and } \\
\text { clinical factors }\end{array}$ & $\begin{array}{l}\text { Descriptive and } \\
\text { inferential } \\
\text { statistics }\end{array}$ & & $\begin{array}{l}\text { The study showed that } \\
\text { visiting clubhouses } \\
\text { appears to be more } \\
\text { beneficial for } \\
\text { improved QOL in a } \\
\text { longer-term } \\
\text { perspective }\end{array}$ \\
\hline $\begin{array}{l}\text { Hultqvist, } \\
\text { Markström [38] }\end{array}$ & $\begin{array}{c}\text { Compared DC and } \\
\text { clubhouses, } \\
\text { concerning the users' } \\
\text { perceptions of unit } \\
\text { and program } \\
\text { characteristics, and } \\
\text { aspects of everyday } \\
\text { occupations in terms } \\
\text { of engagement and } \\
\text { satisfaction }\end{array}$ & Sweden & $\begin{array}{c}\text { Mean age = 48.7; } \\
\text { males and } \\
\text { females }\end{array}$ & $\begin{array}{l}\text { Psychoses, } \\
\text { mood and } \\
\text { anxiety } \\
\text { disorders, } \\
\text { autism/ } \\
\text { neuropsychiatric } \\
\text { disorders }\end{array}$ & $\begin{array}{l}\text { Combined } \\
\text { cross-sectional } \\
\text { and longitudinal } \\
\text { comparative } \\
\text { study/Quantitative }\end{array}$ & $\begin{array}{ll}\text { - } & \text { Evaluation of Perceived } \\
\text { Meaning in Day Centres } \\
\text { (EPM-DC) } \\
\text { - } \quad \text { Productive occupations } \\
\text { (POES-P) } \\
\text { - Satisfaction with Daily } \\
\text { Occupations (SDO) scale }\end{array}$ & $\begin{array}{l}\text { Descriptive and } \\
\text { inferential } \\
\text { statistics }\end{array}$ & & $\begin{array}{l}\text { The users of clubhouse } \\
\text { performed better than } \\
\text { day centre users } \\
\text { various on social } \\
\text { network sub-scales } \\
\text { (feeling valued by } \\
\text { others, feelings of } \\
\text { inclusion and } \\
\text { belonging to a group) }\end{array}$ \\
\hline $\begin{array}{c}\text { Iancu, } \\
\text { Zweekhorst [39] }\end{array}$ & $\begin{array}{l}\text { Analysed and } \\
\text { compared experiences } \\
\text { of recovery on } \\
\text { prevocational services, } \\
\text { in order to assess if } \\
\text { users make progress } \\
\text { towards recovery, } \\
\text { relative to a staged } \\
\text { recovery model }\end{array}$ & Netherlands & $\begin{array}{c}\text { Mean age }=42.5 \\
\text { males and } \\
\text { females }\end{array}$ & $\begin{array}{l}\text { Schizophrenia } \\
\text { and personality } \\
\text { disorders, } \\
\text { depressive and } \\
\text { anxiety } \\
\text { disorders }\end{array}$ & $\begin{array}{c}\text { Interpretive phe- } \\
\text { nomenological } \\
\text { de- } \\
\text { sign/Qualitative }\end{array}$ & - Semi-structured interviews & $\begin{array}{l}\text { Thematic } \\
\text { analysis }\end{array}$ & $\mathrm{H}$ & $\begin{array}{l}\text { The prevocational } \\
\text { services provide the } \\
\text { needed services for } \\
\text { people with mental } \\
\text { disorders who desire } \\
\text { to engage in recovery } \\
\text { (create strong internal } \\
\text { motivation for change) }\end{array}$ \\
\hline
\end{tabular}


Table 2. Cont.

\begin{tabular}{|c|c|c|c|c|c|c|c|c|c|}
\hline Papers & Objectives & Setting & Age/Gender & Participants & $\begin{array}{c}\text { Study De- } \\
\text { sign/Methods }\end{array}$ & Data Collection Instrument & Analysis & $\mathrm{Q}^{+}$ & Summary of Article \\
\hline $\begin{array}{c}\text { Iwasaki, Coyle } \\
{[40]}\end{array}$ & $\begin{array}{l}\text { The role of } \\
\text { leisure-generated } \\
\text { meanings (LGMs) } \\
\text { experienced by } \\
\text { culturally diverse } \\
\text { individuals with } \\
\text { mental illness in } \\
\text { potentially helping } \\
\text { them to better cope } \\
\text { with stress, adjust and } \\
\text { recover }\end{array}$ & USA & $\begin{array}{c}\text { Mean age }=48 \\
\text { males and } \\
\text { females }\end{array}$ & $\begin{array}{c}\text { Bipolar disorder, } \\
\text { major } \\
\text { depression, } \\
\text { schizophrenia, } \\
\text { bipo- } \\
\text { lar/schizophrenic, } \\
\text { schizoaffective } \\
\text { disorder, } \\
\text { substance abuse, } \\
\text { panic disorder, } \\
\text { borderline } \\
\text { personality }\end{array}$ & $\begin{array}{l}\text { Cross-sectional/ } \\
\text { Quantitative }\end{array}$ & $\begin{array}{ll}\text { - } & \text { Colorado Symptom Index } \\
& \text { (CSI } \\
\text { - } & \text { Recovery Assessment Scale } \\
& \text { (RAS } \\
\text { - } & \text { Psychosocial Adjustment to } \\
& \text { Illness Scale (PAIS } \\
-\quad & \text { Leisure Meanings Gained } \\
& \text { Scale (LMGS } \\
\text { - } \quad \text { Leisure Coping Scale (LCS } & \text { Leisure Satisfaction Scale } \\
\text { - } & \text { (LSS } \\
\text { - } \quad \text { Leisure Boredom Scale (LBS } & \text { Perceived Active Living Scale } \\
& \text { (PALS) }\end{array}$ & $\begin{array}{l}\text { Descriptive and } \\
\text { inferential } \\
\text { statistics }\end{array}$ & $\mathrm{H}$ & $\begin{array}{l}\text { Leisure can contribute } \\
\text { to stress-coping, } \\
\text { recovery, adjustment, } \\
\text { and active living for } \\
\text { individuals with } \\
\text { mental illness }\end{array}$ \\
\hline $\begin{array}{c}\text { Ketch, Rubin } \\
\text { [41] }\end{array}$ & $\begin{array}{c}\text { Art appreciation for } \\
\text { veterans with severe } \\
\text { mental illness in a VA } \\
\text { Psychosocial } \\
\text { Rehabilitation and } \\
\text { Recovery Centre }\end{array}$ & USA & & & $\begin{array}{c}\text { Quasi- } \\
\text { experimental } \\
\text { de- } \\
\text { sign/Qualitative }\end{array}$ & - Photos and interviews & $\begin{array}{l}\text { Thematic } \\
\text { analysis }\end{array}$ & $\mathrm{H}$ & $\begin{array}{l}\text { The program had } \\
\text { positive effects on } \\
\text { mood, self-esteem, } \\
\text { socialization } \\
\text { community } \\
\text { participation and } \\
\text { recovery process of } \\
\text { veterans with SMI }\end{array}$ \\
\hline $\begin{array}{c}\text { Kilian, Lauber } \\
\text { [42] }\end{array}$ & $\begin{array}{l}\text { Analyses the } \\
\text { relationships between } \\
\text { employment hours, } \\
\text { psychopathological } \\
\text { symptoms and the } \\
\text { days of inpatient } \\
\text { treatment detected }\end{array}$ & $\begin{array}{l}\text { Six European } \\
\text { centres }\end{array}$ & $\begin{array}{c}\text { Mean age }=37.8 \\
\text { males and } \\
\text { females }\end{array}$ & $\begin{array}{l}\text { Schizophrenia/ } \\
\text { schizoaffective } \\
\text { disorders, } \\
\text { bipolar disorder }\end{array}$ & $\begin{array}{l}\text { Randomized } \\
\text { controlled } \\
\text { trial/Quantitative }\end{array}$ & $\begin{array}{ll}\text { - } & \text { Positive and Negative } \\
\text { - } & \text { Symptoms Scale (PANSS) } \\
\text { OPCRIT }\end{array}$ & $\begin{array}{l}\text { Descriptive and } \\
\text { inferential } \\
\text { statistics }\end{array}$ & $\mathrm{M}$ & $\begin{array}{l}\text { IPS intervention } \\
\text { through its effect on } \\
\text { the time spent in } \\
\text { competitive } \\
\text { employment leads to } \\
\text { reduced need for } \\
\text { psychiatric inpatient } \\
\text { care }\end{array}$ \\
\hline $\begin{array}{c}\text { Koletsi, } \\
\text { Niersman [43] }\end{array}$ & $\begin{array}{l}\text { Explore clients' } \\
\text { experiences of the } \\
\text { support received from } \\
\text { their IPS or vocational } \\
\text { service workers and } \\
\text { the perceived impact } \\
\text { of work on clients' } \\
\text { lives }\end{array}$ & $\begin{array}{l}\text { Six European } \\
\text { centres }\end{array}$ & $\begin{array}{l}\text { Age range = } \\
18-57 ; \text { males } \\
\text { and females }\end{array}$ & $\begin{array}{l}\text { Schizophrenia/ } \\
\text { schizoaffective } \\
\text { disorders, } \\
\text { bipolar disorder }\end{array}$ & $\begin{array}{c}\text { Randomized } \\
\text { controlled } \\
\text { trial/Qualitative }\end{array}$ & - Semi-structured interviews & $\begin{array}{l}\text { Thematic } \\
\text { analysis }\end{array}$ & $\mathrm{H}$ & $\begin{array}{l}\text { The IPS program } \\
\text { improved financial } \\
\text { stability, illness, social } \\
\text { life, increased } \\
\text { self-esteem, } \\
\text { integration into society, } \\
\text { self-improvement, } \\
\text { coping strategy and } \\
\text { reduced loneliness }\end{array}$ \\
\hline
\end{tabular}


Table 2. Cont.

\begin{tabular}{|c|c|c|c|c|c|c|c|c|c|}
\hline Papers & Objectives & Setting & Age/Gender & Participants & $\begin{array}{c}\text { Study De- } \\
\text { sign/Methods }\end{array}$ & Data Collection Instrument & Analysis & $\mathrm{Q}^{+}$ & Summary of Article \\
\hline Lee, Liem [44] & $\begin{array}{l}\text { Explored the } \\
\text { effectiveness of } \\
\text { Assertive Community } \\
\text { Treatment (ACT) for } \\
\text { severely ill mental } \\
\text { patients during a } \\
\text { period of rapid } \\
\text { deinstitutionalization } \\
\text { in Hong Kong }\end{array}$ & Hong Kong & $\begin{array}{l}\text { Age range = } \\
18-65 ; \text { males } \\
\text { and females }\end{array}$ & $\begin{array}{l}\text { Psychotic } \\
\text { disorders }\end{array}$ & $\begin{array}{c}\text { Flanking } \\
\text { historical } \\
\text { control de- } \\
\text { sign/Quantitative }\end{array}$ & $\begin{array}{ll}\text { - } & \text { World Health Organization } \\
\text { Quality of Life (WHOQOL-) } \\
\text { Hong Kong Chinese Version } \\
\text { Brief Psychiatric Rating Scale } \\
\text { (BPRS) } \\
\text { - Clinical Data Analysis and } \\
\text { Reporting System (CDARS) }\end{array}$ & $\begin{array}{l}\text { Descriptive and } \\
\text { inferential } \\
\text { Statistics }\end{array}$ & $\mathrm{H}$ & $\begin{array}{l}\text { The ACT had positive } \\
\text { effect over and above } \\
\text { the conventional } \\
\text { treatment } \\
\text { models—outcome } \\
\text { parameters (bed days, } \\
\text { readmission episodes, } \\
\text { missed psychiatric } \\
\text { appointments, BPRS } \\
\text { and quality of life) } \\
\text { improved }\end{array}$ \\
\hline $\begin{array}{l}\text { Lindstrom, } \\
\text { Hariz [45] }\end{array}$ & $\begin{array}{l}\text { To evaluate clients' } \\
\text { activities of daily } \\
\text { living (ADL) ability } \\
\text { and health factors } \\
\text { outcomes following } \\
\text { their participation in } \\
\text { occupation-centred } \\
\text { interventions in home } \\
\text { and community } \\
\text { settings }\end{array}$ & Sweden & $\begin{array}{l}\text { Mean age }=48 \\
\text { males and } \\
\text { females }\end{array}$ & $\begin{array}{l}\text { Schizophrenia, } \\
\text { schizoaffective } \\
\text { disorder, bipolar } \\
\text { disorder, } \\
\text { Asperger } \\
\text { syndrome, } \\
\text { obsessive } \\
\text { compulsive } \\
\text { disorder }\end{array}$ & $\begin{array}{c}\text { Quasi- } \\
\text { experimental } \\
\text { de- } \\
\text { sign/Quantitative }\end{array}$ & $\begin{array}{ll}\text { - } & \text { Goal Attainment Scaling } \\
& \text { (GAS) } \\
\text { - } & \text { Assessment of Motor and } \\
& \text { Process Skills (AMPS) } \\
\text { - } & \text { Assessment of Social } \\
& \text { Interaction (Swedish version } \\
& \text { BSI-II) } \\
\text { - } & \text { Satisfaction with Daily } \\
& \text { Occupations (SDO) } \\
\text { - } & \text { ADL taxonomy } \\
\text { - } & \text { Symptom Checklist-90 } \\
& \text { (SCL-90) }\end{array}$ & $\begin{array}{l}\text { Descriptive and } \\
\text { inferential } \\
\text { Statistics }\end{array}$ & $\mathrm{H}$ & $\begin{array}{l}\text { The occupational } \\
\text { therapy services } \\
\text { integrated in to } \\
\text { sheltered or supported } \\
\text { housing achieved } \\
\text { positive lifestyle, } \\
\text { meaningful } \\
\text { occupations and } \\
\text { participation in society }\end{array}$ \\
\hline $\begin{array}{l}\text { Lopez-Navarro, } \\
\text { Del Canto [46] }\end{array}$ & $\begin{array}{l}\text { The effectiveness of } \\
\text { group } \\
\text { mindfulness-based } \\
\text { intervention (MBI) in } \\
\text { patients diagnosed } \\
\text { with severe mental } \\
\text { illness }\end{array}$ & $\begin{array}{l}\text { Balearic } \\
\text { Islands }\end{array}$ & $\begin{array}{c}\text { Mean age }= \\
38.44\end{array}$ & $\begin{array}{l}\text { Schizophrenia, } \\
\text { bipolar disorder, } \\
\text { delusional } \\
\text { disorder }\end{array}$ & $\begin{array}{l}\text { Randomized } \\
\text { controlled } \\
\text { trial/Quantitative }\end{array}$ & $\begin{array}{ll}\text { - } & \text { World Health Organization } \\
\text { Quality of Life-BREF } \\
\text { (WHOQOL-BREF) } \\
\text { - } \quad \text { Positive and Negative } \\
\text { Syndrome Scale (PANSS) } \\
\text { - } \quad \text { Mindfulness Attention } \\
\text { Awareness Scale (MAAS) }\end{array}$ & $\begin{array}{l}\text { Descriptive and } \\
\text { inferential } \\
\text { statistics }\end{array}$ & $\mathrm{H}$ & $\begin{array}{l}\text { Mindfulness } \\
\text { intervention in } \\
\text { rehabilitation has } \\
\text { potential to enhance } \\
\text { quality of life and } \\
\text { reduce negative } \\
\text { symptoms }\end{array}$ \\
\hline
\end{tabular}


Table 2. Cont.

\begin{tabular}{|c|c|c|c|c|c|c|c|c|c|}
\hline Papers & Objectives & Setting & Age/Gender & Participants & $\begin{array}{l}\text { Study De- } \\
\text { sign/Methods }\end{array}$ & Data Collection Instrument & Analysis & $\mathrm{Q}^{+}$ & Summary of Article \\
\hline Luk [47] & $\begin{array}{l}\text { Investigate the } \\
\text { long-term effects of a } \\
\text { holistic care program } \\
\text { for the rehabilitation } \\
\text { of persons with } \\
\text { serious mental illness }\end{array}$ & Hong Kong & $\begin{array}{c}\text { Mean age }=35 \\
\text { males and } \\
\text { females }\end{array}$ & $\begin{array}{l}\text { Schizophrenia, } \\
\text { manic } \\
\text { depressive, } \\
\text { depression }\end{array}$ & $\begin{array}{l}\text { Cross-sectional/ } \\
\text { Quantitative }\end{array}$ & $\begin{array}{ll}\text { - } & \text { World Health Organization } \\
& \text { Quality of Life Measure } \\
& \text { (WHOQOL-BREF(HK) } \\
\text { - } & \text { General Health } \\
& \text { Questionnaire (GHQ) } \\
\text { - } & \text { Rosenberg Self-Esteem Scale } \\
\text { (ESTEEM) } \\
\text { - } \quad \text { Social Support } \\
\text { - } \\
\text { Questionnaire-6 (SSQ-6) } \\
\text { Purpose in Life } \\
\text { - } \quad \text { Questionnaire (PIL) } \\
\text { - } & \text { Sopelessness Scale (HOPE) } \\
\text { SSQ-Satisfaction }\end{array}$ & $\begin{array}{l}\text { Descriptive and } \\
\text { inferential } \\
\text { statistics }\end{array}$ & $\mathrm{H}$ & $\begin{array}{l}\text { The program is } \\
\text { effective to provide } \\
\text { positive } \\
\text { changes-support, } \\
\text { encouragement, } \\
\text { self-confidence, } \\
\text { spiritual assistance } \\
\text { and reflection of } \\
\text { values }\end{array}$ \\
\hline $\begin{array}{l}\text { Malinovsky, } \\
\text { Lehrer [48] }\end{array}$ & $\begin{array}{l}\text { Evaluate the } \\
\text { effectiveness of a } \\
\text { recovery-oriented } \\
\text { transformation carried } \\
\text { out by a large, private, } \\
\text { not-for-profit } \\
\text { psychiatric } \\
\text { rehabilitation } \\
\text { organization serving } \\
\text { individuals with SM }\end{array}$ & USA & $\begin{array}{c}\text { Mean age }= \\
\text { 46.42; males and } \\
\text { females }\end{array}$ & $\begin{array}{l}\text { Schizophrenia, } \\
\text { mood disorder } \\
\text { (unipo- } \\
\text { lar/bipolar), } \\
\text { other psychotic } \\
\text { disorder }\end{array}$ & $\begin{array}{l}\text { Longitudinal } \\
\text { study/Quantitative }\end{array}$ & $\begin{array}{ll}\text { - } & \text { Multnomah Community } \\
& \text { Ability Scale-Revised } \\
& \text { Clinician Rated (MCAS-R) } \\
\text { - } & \text { Self-Report (MCAS-SR) } \\
\text { - } & \text { Competency Assessment } \\
\text { - } & \text { Instrument (CAI) } \\
\text { - } & \text { Client Hope Scale (SHS) } \\
& \text { Therapist Version (WAI-T) }\end{array}$ & $\begin{array}{l}\text { Descriptive and } \\
\text { inferential } \\
\text { statistics }\end{array}$ & $\mathrm{H}$ & $\begin{array}{c}\text { Recovery-oriented } \\
\text { services are effective } \\
\text { to reduce } \\
\text { hospitalizations and } \\
\text { improve quality of life }\end{array}$ \\
\hline $\begin{array}{c}\text { Mizock, } \\
\text { Russinova [49] }\end{array}$ & $\begin{array}{l}\text { Describe the } \\
\text { development and } \\
\text { feasibility of the } \\
\text { recovery narrative } \\
\text { photovoice } \\
\text { intervention }\end{array}$ & USA & & $\begin{array}{l}\text { Serious mental } \\
\text { illnesses }\end{array}$ & $\begin{array}{l}\text { Community- } \\
\text { based } \\
\text { participatory re- } \\
\text { search/Quantitative }\end{array}$ & $\begin{array}{ll}- & \text { Ryff Scale of Psychological } \\
& \text { Well-Being } \\
- & \text { Empowerment Scale } \\
\text { e } & \text { Community Integration } \\
& \text { Measure }\end{array}$ & $\begin{array}{l}\text { Descriptive } \\
\text { statistics }\end{array}$ & & $\begin{array}{l}\text { The program has the } \\
\text { potential to facilitate } \\
\text { recovery-related } \\
\text { outcomes, including } \\
\text { empowerment, } \\
\text { positive identity, and } \\
\text { community } \\
\text { integration }\end{array}$ \\
\hline $\begin{array}{c}\text { Mizock, } \\
\text { Russinova [50] }\end{array}$ & $\begin{array}{l}\text { Explore the meaning } \\
\text { of recovery for } \\
\text { individuals with } \\
\text { serious mental illness }\end{array}$ & USA & & $\begin{array}{l}\text { Serious mental } \\
\text { illness }\end{array}$ & $\begin{array}{l}\text { Community- } \\
\text { based } \\
\text { participatory re- } \\
\text { search/Qualitative }\end{array}$ & - Photos and archival data & $\begin{array}{l}\text { Thematic } \\
\text { analysis }\end{array}$ & $\mathrm{H}$ & $\begin{array}{l}\text { The study identify } \\
\text { several internal and } \\
\text { external recovery } \\
\text { strategies and } \\
\text { outcomes }\end{array}$ \\
\hline
\end{tabular}


Table 2. Cont.

\begin{tabular}{|c|c|c|c|c|c|c|c|c|c|}
\hline Papers & Objectives & Setting & Age/Gender & Participants & $\begin{array}{c}\text { Study De- } \\
\text { sign/Methods }\end{array}$ & Data Collection Instrument & Analysis & $\mathrm{Q}^{+}$ & Summary of Article \\
\hline $\begin{array}{c}\text { Panczak and } \\
\text { Pietkiewicz [51] }\end{array}$ & $\begin{array}{l}\text { Explore personal } \\
\text { experiences of people } \\
\text { employed in } \\
\text { Vocational } \\
\text { Development Centres }\end{array}$ & Poland & $\begin{array}{l}\text { Age range }= \\
28-58 ; \text { males } \\
\text { and females }\end{array}$ & $\begin{array}{l}\text { Schizophrenia } \\
\text { spectrum } \\
\text { disorders }\end{array}$ & $\begin{array}{l}\text { Interpretative } \\
\text { phenomenologi- } \\
\text { cal } \\
\text { de- } \\
\text { sign/Qualitative }\end{array}$ & - Semi-structured interviews & $\begin{array}{l}\text { Consecutive } \\
\text { analytical }\end{array}$ & $\mathrm{H}$ & $\begin{array}{l}\text { The program } \\
\text { improved the } \\
\text { economic and social } \\
\text { well-being of people } \\
\text { with schizophrenia- } \\
\text { economic } \\
\text { empowerment, } \\
\text { empowerment, } \\
\text { functioning and social } \\
\text { inclusion }\end{array}$ \\
\hline $\begin{array}{c}\text { Raeburn, } \\
\text { Schmied [52] }\end{array}$ & $\begin{array}{l}\text { Explore how recovery } \\
\text { practices are } \\
\text { implemented in a } \\
\text { psychosocial } \\
\text { clubhouse }\end{array}$ & Australia & $\begin{array}{l}\text { Mean age }=47 \\
\text { males and } \\
\text { females }\end{array}$ & $\begin{array}{c}\text { Schizophrenia, } \\
\text { bipolar disorder } \\
\text { or } \\
\text { schizoaffective } \\
\text { disorder }\end{array}$ & $\begin{array}{c}\text { Case } \\
\text { study/Qualitative }\end{array}$ & $\begin{array}{ll}- & \text { In-depth interviews (IDIs) } \\
\text { Observations-Spradley's } \\
\text { field note domains, and the } \\
\text { Recovery and Promotion } \\
\text { Fidelity Scale (RPFS) }\end{array}$ & $\begin{array}{l}\text { Theoretical } \\
\text { thematic } \\
\text { analysis }\end{array}$ & $\mathrm{H}$ & $\begin{array}{l}\text { The psychosocial } \\
\text { clubhouse is a } \\
\text { community that } \\
\text { provide opportunity } \\
\text { to participate in a } \\
\text { personal recovery } \\
\text { journey }\end{array}$ \\
\hline $\begin{array}{c}\text { Salyers, } \\
\text { McGuire [53] }\end{array}$ & $\begin{array}{c}\text { To rigorously test } \\
\text { Illness Management } \\
\text { and Recovery (IMR) } \\
\text { against an active } \\
\text { control group in a } \\
\text { sample that included } \\
\text { veterans }\end{array}$ & USA & $\begin{array}{c}\text { Mean age }=47.7 \\
\text { males and } \\
\text { females }\end{array}$ & $\begin{array}{l}\text { Schizophrenia, } \\
\text { schizoaffective } \\
\text { disorder }\end{array}$ & $\begin{array}{l}\text { Randomized } \\
\text { controlled } \\
\text { trial/Quantitative }\end{array}$ & $\begin{array}{ll}\text { - } & \text { Structured Clinical Interview } \\
& \text { for DSM-IV } \\
\text { - } & \text { Positive and Negative } \\
& \text { Syndrome Scale (PANSS) } \\
\text { - } & \text { Quality of Life Scale (QLS) } \\
\text { - } & \text { Patient Activation Measure } \\
\text { - } & \text { Morisky Scale } \\
\text { - } & \text { Recovery Assessment Scale } \\
& \text { (RAS) } \\
\text { - } & \text { State Hope Scale }\end{array}$ & $\begin{array}{l}\text { Descriptive and } \\
\text { inferential } \\
\text { statistics }\end{array}$ & $\mathrm{H}$ & $\begin{array}{l}\text { Improved significantly } \\
\text { in a number of } \\
\text { domains related to } \\
\text { illness management- } \\
\text { symptoms, } \\
\text { psychosocial } \\
\text { functioning, self-rated } \\
\text { illness management, } \\
\text { and emergency } \\
\text { department use }\end{array}$ \\
\hline $\begin{array}{l}\text { Svanberg, } \\
\text { Gumley [54] }\end{array}$ & $\begin{array}{l}\text { Explore the experience } \\
\text { of recovery from } \\
\text { mental illness in the } \\
\text { context of two } \\
\text { emerging social firms }\end{array}$ & Scotland & $\begin{array}{l}\text { Age range }= \\
\text { 19-64; males } \\
\text { and females }\end{array}$ & $\begin{array}{c}\text { Bipolar disorder, } \\
\text { depression, } \\
\text { psychosis, } \\
\text { anxiety, } \\
\text { addictions }\end{array}$ & $\begin{array}{l}\text { Social } \\
\text { constructionist } \\
\text { (grounded the- } \\
\text { ory)/Qualitative }\end{array}$ & $\begin{array}{l}\text { - Open-ended interview } \\
\text { questions }\end{array}$ & $\begin{array}{l}\text { Thematic } \\
\text { analysis }\end{array}$ & $\mathrm{H}$ & $\begin{array}{l}\text { The social firms are } \\
\text { effective to enhance } \\
\text { self-confidence, } \\
\text { acceptance and } \\
\text { inclusion of people } \\
\text { with mental illness }\end{array}$ \\
\hline
\end{tabular}


Table 2. Cont.

\begin{tabular}{|c|c|c|c|c|c|c|c|c|c|}
\hline Papers & Objectives & Setting & Age/Gender & Participants & $\begin{array}{c}\text { Study De- } \\
\text { sign/Methods }\end{array}$ & Data Collection Instrument & Analysis & $\mathrm{Q}^{+}$ & Summary of Article \\
\hline $\begin{array}{l}\text { Swildens, van } \\
\text { Busschbach [55] }\end{array}$ & $\begin{array}{l}\text { Investigate the effect } \\
\text { of the Boston } \\
\text { Psychiatric } \\
\text { Rehabilitation (PR) } \\
\text { approach on } \\
\text { attainment of personal } \\
\text { rehabilitation goals, } \\
\text { social functioning, } \\
\text { empowerment, needs } \\
\text { for care, and quality of } \\
\text { life in people with } \\
\text { severe mental illness } \\
\text { (SMI) in the } \\
\text { Netherlands }\end{array}$ & Netherlands & $\begin{array}{l}\text { Age }=41 \text { and } \\
\text { over; males and } \\
\text { females }\end{array}$ & $\begin{array}{l}\text { Schizophrenia } \\
\text { or } \\
\text { schizoaffective } \\
\text { disorder, bipolar } \\
\text { disorder, } \\
\text { depressive or } \\
\text { anxiety disorder, } \\
\text { personality, } \\
\text { addiction, } \\
\text { cognitive } \\
\text { disorder }\end{array}$ & $\begin{array}{l}\text { Randomized } \\
\text { controlled } \\
\text { trial/Quantitative }\end{array}$ & $\begin{array}{ll}\text { - } & \text { Self-report Social Functioning } \\
& \text { Scale } \\
\text { - } & \text { Camberwell Assessment of } \\
& \text { Need Short Appraisal } \\
& \text { Schedule } \\
\text { - } & \text { WHOQOL-BREF } \\
\text { - } & \text { Personal Empowerment Scale } \\
\text { - } & \text { BPRS-Extended version } \\
\text { - } \text { GAF-symptoms and } \\
\text { disabilities } \\
\text { Client Socio-Demographic } \\
\text { and Service Receipt } \\
\text { Inventory-European } \\
\text { version } \\
\text { - PR Beliefs, Goals and } \\
\text { Practices scale } \\
\quad \text { Working Alliance Inventory }\end{array}$ & $\begin{array}{l}\text { Descriptive and } \\
\text { inferential } \\
\text { statistics }\end{array}$ & $\mathrm{H}$ & $\begin{array}{c}\text { Psychiatric } \\
\text { rehabilitation has a } \\
\text { significant impact on } \\
\text { goal attainment, } \\
\text { societal participation } \\
\text { and social contacts }\end{array}$ \\
\hline $\begin{array}{c}\text { Tjornstrand, } \\
\text { Bejerholm [56] }\end{array}$ & $\begin{array}{l}\text { Gaining knowledge } \\
\text { regarding the } \\
\text { occupations } \\
\text { performed in day } \\
\text { centres, in terms of the } \\
\text { participants' } \\
\text { descriptions of what } \\
\text { they were doing }\end{array}$ & Sweden & $\begin{array}{l}\text { Mean age }=45.3 \\
\text { males and } \\
\text { females }\end{array}$ & $\begin{array}{l}\text { Schizophrenia, } \\
\text { other psychoses }\end{array}$ & $\begin{array}{c}\text { Interpretative } \\
\text { phenomenologi- } \\
\text { cal } \\
\text { de- } \\
\text { sign/Qualitative }\end{array}$ & $\begin{array}{ll}-\quad & \text { Time-use diary } \\
-\quad & \text { Profiles of Occupational } \\
& \text { Engagement POES }\end{array}$ & Content analysis & $\mathrm{H}$ & $\begin{array}{l}\text { The study showed that } \\
\text { social interaction and } \\
\text { occupations formed } \\
\text { the two foundations of } \\
\text { the day centres }\end{array}$ \\
\hline Tsang, Ng [57] & $\begin{array}{l}\text { Effects of the } \\
\text { 'clubhouse' model of } \\
\text { rehabilitation on } \\
\text { various psychosocial } \\
\text { issues for Chinese } \\
\text { patients with } \\
\text { schizophrenia living in } \\
\text { the community }\end{array}$ & Hong Kong & $\begin{array}{l}\text { Mean age }=40.5 \\
\text { males and } \\
\text { females }\end{array}$ & $\begin{array}{c}\text { Chronic } \\
\text { schizophrenia }\end{array}$ & $\begin{array}{l}\text { longitudinal, } \\
\text { case-control and } \\
\text { naturalistic de- } \\
\text { sign/Quantitative }\end{array}$ & $\begin{array}{ll}\text { - } & \text { Demographic and clinical } \\
\text { - } & \text { variables } \\
& \text { Positive and Negative } \\
\text { - } & \text { Syndrome Scale } \\
\text { - } & \text { Beck Depression Inventory } \\
\text { - } & \text { Quality of Life-Brief Version } \\
\text { - } \quad \text { Rosenberg Self-Esteem Scale } \\
\quad \text { Levenson Internality, } \\
\quad \text { Powerful Others and Chance } \\
\text { Scale of Locus of Control }\end{array}$ & $\begin{array}{l}\text { Descriptive and } \\
\text { inferential } \\
\text { statistics }\end{array}$ & & $\begin{array}{l}\text { The program } \\
\text { improved the } \\
\text { psychological, social } \\
\text { relationship and } \\
\text { environmental quality } \\
\text { of life of participants }\end{array}$ \\
\hline
\end{tabular}


Table 2. Cont

\begin{tabular}{|c|c|c|c|c|c|c|c|c|c|}
\hline Papers & Objectives & Setting & Age/Gender & Participants & $\begin{array}{l}\text { Study De- } \\
\text { sign/Methods }\end{array}$ & Data Collection Instrument & Analysis & $\mathrm{Q}^{+}$ & Summary of Article \\
\hline $\begin{array}{c}\text { Tondora, } \\
\text { O'Connell [58] }\end{array}$ & $\begin{array}{c}\text { Rationale, design, and } \\
\text { lessons learned during } \\
\text { the implementation of } \\
\text { a randomized clinical } \\
\text { trial testing the effect } \\
\text { of using peer } \\
\text { facilitative advocates } \\
\text { to promote culturally } \\
\text { responsive } \\
\text { person-centred care } \\
\text { planning on QOL } \\
\text { variables, community } \\
\text { connections, and } \\
\text { coping for people of } \\
\text { colour with psychotic } \\
\text { disorders }\end{array}$ & USA & $\begin{array}{l}\text { Mean age }=43.5 \\
\text { males and } \\
\text { females }\end{array}$ & $\begin{array}{l}\text { Schizophrenia, } \\
\text { schizoaffective } \\
\text { disorder, or } \\
\text { affective } \\
\text { disorder }\end{array}$ & $\begin{array}{l}\text { Randomized } \\
\text { clinical } \\
\text { trial/Quantitative }\end{array}$ & 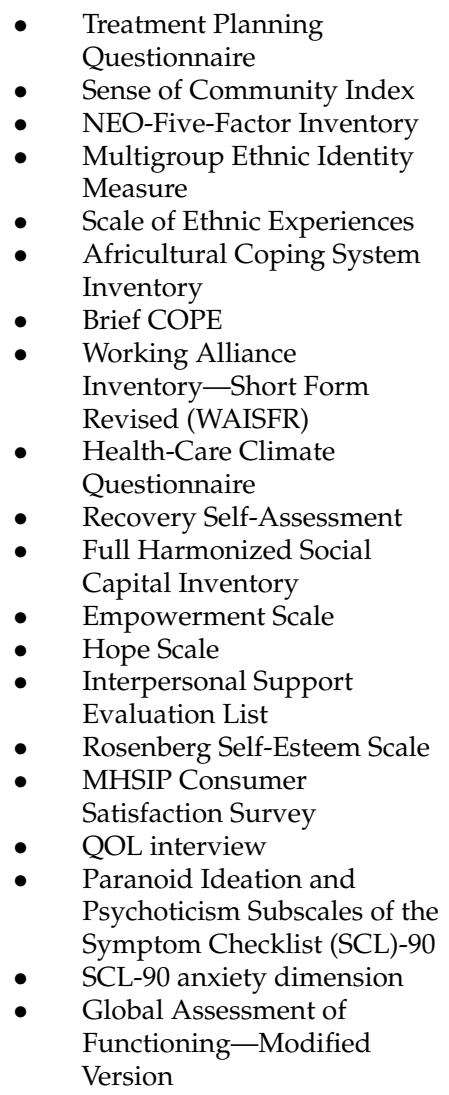 & $\begin{array}{l}\text { Descriptive and } \\
\text { inferential } \\
\text { statistics }\end{array}$ & $\mathrm{H}$ & $\begin{array}{l}\text { The project suggests } \\
\text { the need to make } \\
\text { cultural modifications, } \\
\text { longer engagement } \\
\text { period with } \\
\text { participants }\end{array}$ \\
\hline
\end{tabular}


Table 2. Cont.

\begin{tabular}{|c|c|c|c|c|c|c|c|c|c|}
\hline Papers & Objectives & Setting & Age/Gender & Participants & $\begin{array}{c}\text { Study De- } \\
\text { sign/Methods }\end{array}$ & Data Collection Instrument & Analysis & $\mathrm{Q}^{+}$ & Summary of Article \\
\hline $\begin{array}{c}\text { Twamley, Vella } \\
\text { [59] }\end{array}$ & $\begin{array}{l}\text { To evaluate the } \\
\text { efficacy of supported } \\
\text { employment for } \\
\text { middle-aged or older } \\
\text { people with } \\
\text { schizophrenia }\end{array}$ & USA & $\begin{array}{l}\text { Mean age }=51 \\
\text { males and } \\
\text { females }\end{array}$ & $\begin{array}{l}\text { Schizophrenia } \\
\text { or } \\
\text { schizoaffective } \\
\text { disorder aged }\end{array}$ & $\begin{array}{l}\text { Randomized } \\
\text { controlled } \\
\text { trial/Quantitative }\end{array}$ & $\begin{array}{ll}\text { - } & \text { UCSD Performance-Based } \\
\text { - } & \text { Skills Assessment (UPSA) } \\
& \text { Positive and Negative } \\
\text { - } & \text { Hamdrome Scale (PANSS) } \\
\text { Hamilton Rating Scale for } \\
\text { Depression }\end{array}$ & $\begin{array}{l}\text { Descriptive and } \\
\text { inferential } \\
\text { statistics }\end{array}$ & $\mathrm{H}$ & $\begin{array}{l}\text { Individual Placement } \\
\text { and Support (IPS) was } \\
\text { effective for people } \\
\text { with schizophrenia } \\
\text { compared with } \\
\text { conventional } \\
\text { vocational } \\
\text { rehabilitation (CVR) }\end{array}$ \\
\hline $\begin{array}{c}\text { Whitley, Harris } \\
\text { [61] }\end{array}$ & $\begin{array}{l}\text { Explore and elucidate } \\
\text { whether components } \\
\text { of these communities } \\
\text { appeared to assist } \\
\text { recovery from the } \\
\text { point of view of } \\
\text { consumers, and if so } \\
\text { which were the most } \\
\text { important factors }\end{array}$ & USA & & & $\begin{array}{c}\text { Grounded } \\
\text { theory ap- } \\
\text { proach/Qualitative }\end{array}$ & $\begin{array}{ll}- & \text { Focus groups } \\
- & \text { Observations }\end{array}$ & $\begin{array}{l}\text { Grounded } \\
\text { theory approach }\end{array}$ & $\mathrm{H}$ & $\begin{array}{l}\text { The community is } \\
\text { perceived as a place of } \\
\text { safety, surrogate } \\
\text { family, socialization } \\
\text { and individual growth }\end{array}$ \\
\hline $\begin{array}{l}\text { Zemore and } \\
\text { Kaskutas [62] }\end{array}$ & $\begin{array}{l}\text { explores whether } \\
\text { services received } \\
\text { differed by program } \\
\text { modality (i.e., day } \\
\text { hospital vs. } \\
\text { residential) }\end{array}$ & USA & $\begin{array}{l}\text { Age }=\geq 18 \\
\text { males and } \\
\text { females }\end{array}$ & $\begin{array}{l}\text { Alcohol- } \\
\text { dependent only, } \\
\text { drug-dependent } \\
\text { only, alcohol } \\
\text { and drug } \\
\text { dependent }\end{array}$ & $\begin{array}{l}\text { Randomized } \\
\text { controlled } \\
\text { trial/Quantitative }\end{array}$ & $\begin{array}{ll}\text { - } & \text { Treatment Services Review } \\
\text { (TSR) } \\
\text { - } \quad \begin{array}{l}\text { Demographics and other } \\
\text { covariates }\end{array}\end{array}$ & $\begin{array}{l}\text { Descriptive and } \\
\text { inferential } \\
\text { statistics }\end{array}$ & $\mathrm{H}$ & $\begin{array}{c}\text { Residential } \\
\text { participants showed } \\
\text { greater participation in } \\
\text { sober recreational } \\
\text { events and informal } \\
\text { socialization with } \\
\text { peers. Higher } \\
\text { participation in } \\
\text { optional or } \\
\text { extracurricular 12-step } \\
\text { meetings was } \\
\text { associated with better } \\
\text { treatment outcomes }\end{array}$ \\
\hline
\end{tabular}


Table 2. Cont.

\begin{tabular}{|c|c|c|c|c|c|c|c|c|c|}
\hline Papers & Objectives & Setting & Age/Gender & Participants & $\begin{array}{c}\text { Study De- } \\
\text { sign/Methods }\end{array}$ & Data Collection Instrument & Analysis & $\mathrm{Q}^{+}$ & Summary of Article \\
\hline Zhou, Zhou [63] & $\begin{array}{l}\text { Effectiveness of the } \\
\text { rehabilitation services } \\
\text { provided at the } \\
\text { 'Sunshine Soul Park' } \\
\text { on the psychotic } \\
\text { symptoms and social } \\
\text { functioning of } \\
\text { individuals with } \\
\text { schizophrenia }\end{array}$ & China & $\begin{array}{c}\text { Mean age }=39.2 \\
\text { males and } \\
\text { females }\end{array}$ & Schizophrenia & $\begin{array}{l}\text { Non- } \\
\text { randomized } \\
\text { controlled } \\
\text { trial/Quantitative }\end{array}$ & $\begin{array}{ll}- & \text { PANSS } \\
& \text { Quality of Life Inventory-74 } \\
& \text { (GQOLI-74) } \\
\text { - } & \text { Social Disability Screening } \\
& \text { Schedule (SDSS) } \\
-\quad & \text { Insight and Treatment } \\
& \text { Attitude Questionnaire } \\
& \text { (ITAQ) }\end{array}$ & $\begin{array}{l}\text { Descriptive and } \\
\text { inferential } \\
\text { statistics }\end{array}$ & $\mathrm{H}$ & $\begin{array}{l}\text { The intervention is } \\
\text { effective in improving } \\
\text { the social functioning } \\
\text { of patients with } \\
\text { schizophrenia and in } \\
\text { helping them } \\
\text { understand and } \\
\text { manage their illness }\end{array}$ \\
\hline
\end{tabular}




\section{Context for Implementing Recovery Services}

This review identified five environments or contexts where recovery services are implemented (Table 3). Environments where recovery services were implemented were communities, residential facilities and services via psychiatric hospital and primary health care settings $[25,26,30,31,44,57]$ (Table 3 ). Four papers suggested that recovery services can be offered through home-based care $[33,34,36,45]$ and day centre structure programs $[28,37,56]$.

Table 3. Themes identified from mixed-methods synthesis.

\begin{tabular}{|c|c|c|c|}
\hline Global Themes & Organizing Themes & $\mathbf{N}$ & Papers \\
\hline \multirow{5}{*}{$\begin{array}{l}\text { Context or environment } \\
\text { for implementing recovery } \\
\text { services }\end{array}$} & Community based & 22 & {$[24,27,29,32,37,38,40,45-48,50,51,53-55,57-61,63]$} \\
\hline & Residential facilities & 7 & {$[27-29,35,36,41,62]$} \\
\hline & $\begin{array}{c}\text { Home-based care or } \\
\text { services }\end{array}$ & 4 & {$[33,34,36,45]$} \\
\hline & $\begin{array}{c}\text { Day centre-structured } \\
\text { program }\end{array}$ & 3 & {$[28,37,56]$} \\
\hline & $\begin{array}{c}\text { Psychiatric day } \\
\text { hospital/primary services }\end{array}$ & 6 & {$[25,26,30,31,44,57]$} \\
\hline \multirow{3}{*}{$\begin{array}{l}\text { Mechanisms for } \\
\text { implementing recovery } \\
\text { services }\end{array}$} & $\begin{array}{c}\text { Integrated recovery } \\
\text { services }\end{array}$ & 17 & {$[24-26,30,34,40,44-48,53,56,58,61-63]$} \\
\hline & Vocational rehabilitation & 18 & {$[27-29,33,34,37-39,42,43,51,54-57,59,60]$} \\
\hline & $\begin{array}{c}\text { Recovery narrative } \\
\text { photovoice and art making }\end{array}$ & 5 & {$[32,35,41,49,50]$} \\
\hline \multirow{9}{*}{$\begin{array}{c}\text { Outcome of recovery } \\
\text { services }\end{array}$} & Psychiatric medication & 10 & {$[24,25,33,36,42,44,48,53,62,63]$} \\
\hline & Improving functionality & 14 & {$[24,26,36,37,39,41,43-45,48,51,53,55,63]$} \\
\hline & Reduce symptoms & 11 & {$[25,30,31,36,42,44-46,53,57,63]$} \\
\hline & $\begin{array}{l}\text { Improving physical health } \\
\text { and social behaviour }\end{array}$ & 7 & {$[24,26,36,45,46,48]$} \\
\hline & Economic empowerment & 19 & {$[24-29,31,33,34,36,38,42,43,47,51,56,57,59,60]$} \\
\hline & Household integration & 3 & {$[24,26,36]$} \\
\hline & $\begin{array}{c}\text { Social inclusion } \\
\text { (community integration) }\end{array}$ & 27 & {$[24-26,30-35,37-39,41,43-45,49,51,52,54-58,61-63]$} \\
\hline & Social support & 7 & {$[31,33,34,36,39,50,61]$} \\
\hline & Self-efficacy & 21 & {$[24-26,30,32-35,38-40,43,47,49-52,54,55,57,61]$} \\
\hline
\end{tabular}

\section{Mechanisms for Implementing Recovery Services}

\subsection{An Integrated Recovery Service}

In this review, an integrated recovery service model is described as any services that seek to promote and support restoration, 'remediation and reconnection'. The concept employs both an overarching, inherently collaborative and integrated approach to mental health services. Most of the review papers (16/40) described integrated recovery models as services used to achieve the personal recovery of adults with severe mental illness (Table 3 ). Most of the papers suggested that integrated recovery services can be delivered through illness management [47,48,53,58,61-63], mindfulness-based interventions [46], task-sharing or task-shifting approaches [25,26], home visits [24,40,44], active leisure or recreational activities [34,56] and music therapy [30] (see Table 4). The reviewed papers highlighted that integrated recovery services generally aim at developing independent living skills, improving quality of life, community mobilisation [48], reducing inpatient and crisis services, adhering to treatment and setting meaningful goals towards recovery $[24,53,58]$. 
Integrated recovery services can be offered through training sessions (e.g., hours, days and weekly for several months) $[25,26,30,44,46,53]$. For instance, past study regarding integrated recovery was enhanced with mindfulness group therapy sessions which were run for 60 min throughout 26 weeks [46] (see Table 4). Conversely, music-creation therapy used as a recovery service was run for 32 weekly sessions, with a duration of $90 \mathrm{~min}$ for each session [30]. Generally, the activities of integrated recovery service focused on cognitive behaviour therapy techniques, psychoeducation, relapse prevention, social and coping skills training (meals, guidance in activities of daily living, role-playing, hobby groups) [24,47,53], adherence support, family therapy, crisis management, household contribution and understanding medication [24,25,44]. More specifically, Tjornstrand, Bejerholm [56] recommended that active leisure implemented as recovery services can include activities-playing sports; the opportunity to play games, eat, and socialize; embarking on excursions; relaxation (see Table 4).

Two studies concluded that conventional rehabilitation services can incorporate additional innovative interventions aimed to achieve recovery for consumers [30,47]. For instance, Luk [47] recommended the inclusion of spiritual intervention (a form of hymn singing, Bible reading, personal sharing and intercessions) in conventional rehabilitation services. Similarly, Chang, Chen [30] recommended the use of music-creation therapy as a recovery service for adults with SMI. These recovery services are delivered by different mental health professionals, including clinical psychologists, community health workers, psychiatrists, occupational therapists, social workers and counsellors [25,26,40,44,47]. Some studies further suggested that non-specialists are sometimes trained to deliver recovery services through task-sharing or task-shifting approaches. Some of the non-specialist professionals are auxiliary social workers $[25,47]$.

\subsection{Vocational Rehabilitation (Individual Placement Services)}

Eighteen of the included papers recommended several vocational rehabilitation programs used to improving the lives of consumers (see Table 3). These are Individual Placement and Support (IPS) $[28,29,33,34,43,57,59,60]$, supported employment enterprises, sheltered employment $[27,34,51,57]$, conventional vocational rehabilitation [59] and social firms [51,54].

Most of the included papers described the process of implementing Individual Placement and Support. The papers suggested that the IPS is implemented through phases such as initial vocational assessments (e.g., to identify clients' strengths and work skills), job searching (e.g., searching job sites, applying for work online and accompany clients to interviews), individual job development (e.g., intensive supervision), work performance monitoring, support for employers and continuing post-employment support for clients $[27-29,33,59]$. In addition, some papers recommended that Individual Placement and Support workers receive training and regular supervision to provide effective services $[29,60]$ (see Table 4). IPS employment can take several forms, including services (e.g., cleaning, gardening, catering, clerical and administrative work) [56,57], training clients for the labour market, agricultural production and creative projects (e.g., painting, drawing, sculpture, ceramics and textiles, assembly lines, carpentry shops, computer repair centres, bicycle repair shops woodworking and furniture making) $[39,54]$.

\subsection{Recovery Narrative Photovoice, Art Making and Exhibition}

Five of the included papers recommended photovoice, art making and exhibition as interventions used to construct recovery $[32,35,41,49,50]$. Photovoice, art making and exhibition are used to achieve recovery, empowerment, community integration $[32,49,50]$ and share difficult experiences non-verbally [35]. The intervention aims to explore, document and share ideas about recovery. It involves the construction of text with photographs through the exhibition and large group discussion [32,49,50]. More importantly, the intervention helps to avoid the stigma that is associated with the conventional process of delivering mental health services [41]. For instance, Clements [32] suggested that readers 
or audience of photovoice interventions become part of the construction of the meaning of recovery. The intervention allows people with serious mental illness to communicate their needs and ideas to the public, as well as to policy makers.

Photovoice, art and exhibition interventions are delivered through weekly class sessions and community outings $[41,49,50]$ (Table 3 ). The content of the class sessions focus on writing exercises, psychoeducational handouts, and activities on how to construct empowering narratives of recovery and stigmatization [49,50] (see Table 4). In addition, Ketch, Rubin [41] suggested that the class sessions begin with sharing previous experiences or knowledge about art making. The final outcomes of the photovoice, art and exhibition interventions are documented through the final recovery photo gallery, text pieces, art shows, public exhibition, creative arts (e.g., painting, ceramics, silk screening, and mosaics) and displays of art prints $[32,35,41,50]$.

Table 4. Mechanisms for implementing recovery services.

\begin{tabular}{|c|c|c|c|}
\hline Recovery Services & Intervention & How to Deliver the Intervention & Process or Outcome \\
\hline & Illness management & $\begin{array}{l}\text { Rehabilitation training (hourly, days and } \\
\text { weekly) meetings for adult living with } \\
\text { severe mental illness and family members } \\
\text { Day treatment, medication monitoring and } \\
\text { intellectual activities } \\
\text { - Cognitive behaviour therapy techniques, } \\
\text { psychoeducation, relapse prevention, social } \\
\text { skills training, spiritual intervention } \\
\text { Inclusion of spiritual intervention in } \\
\text { - } \quad \text { Attending didactic and counselling sessions }\end{array}$ & $\begin{array}{l}\text { Antipsychotic medication } \\
\text { adherence, improve } \\
\text { knowledge, decrease } \\
\text { relapse, reducing inpatient } \\
\text { and crisis services }\end{array}$ \\
\hline & $\begin{array}{l}\text { Mindfulness-based } \\
\text { interventions }\end{array}$ & $\begin{array}{l}\text { - Mindfulness group therapy sessions } \\
\text { (training hourly, daily and weekly) } \\
\text { Meetings for consumers and family } \\
\text { members }\end{array}$ & $\begin{array}{l}\text { Improving functioning, } \\
\text { symptoms and quality of } \\
\text { life }\end{array}$ \\
\hline \multirow[t]{3}{*}{$\begin{array}{l}\text { Integrated recovery } \\
\text { services }\end{array}$} & $\begin{array}{c}\text { Task-sharing or } \\
\text { task-shifting approaches }\end{array}$ & $\begin{array}{l}\text { - Participatory training for non-specialists to } \\
\text { provide mental health services in } \\
\text { communities } \\
\text { - Refer adults living with severe mental } \\
\text { illness to primary health care and specialist } \\
\text { services }\end{array}$ & $\begin{array}{l}\text { Increase access to } \\
\text { psychiatric medication, } \\
\text { improve physical health } \\
\text { and social behaviour, } \\
\text { self-efficacy }\end{array}$ \\
\hline & Home visits concept & $\begin{array}{l}\text { Weekly home visits by mental health } \\
\text { professionals to deliver mental health } \\
\text { education, advocacy, community outreach } \\
\text { and community orientation } \\
\text { Adults living with severe mental illness } \\
\text { and family members are taught about } \\
\text { where and how to buy medication } \\
\text { Reminding consumers and families to } \\
\text { attend follow up }\end{array}$ & $\begin{array}{l}\text { Household integration, } \\
\text { improving self-care or } \\
\text { practical skills }\end{array}$ \\
\hline & Music-creation therapy & $\begin{array}{l}\text { - A } 90 \text { min music-creation group activity } \\
\text { organized weekly for } 32 \text { consecutive weeks }\end{array}$ & $\begin{array}{l}\text { Community acceptance, } \\
\text { inclusion, empowerment }\end{array}$ \\
\hline
\end{tabular}


Table 4. Cont.

\begin{tabular}{|c|c|c|c|}
\hline Recovery Services & Intervention & How to Deliver the Intervention & Process or Outcome \\
\hline & $\begin{array}{l}\text { Active leisure or } \\
\text { recreational activities }\end{array}$ & $\begin{array}{l}\text { - Playing sports, opportunity to play games, } \\
\text { eat, and socialize, embarking on excursions and } \\
\text { relaxation }\end{array}$ & $\begin{array}{c}\text { Social inclusion } \\
\text { (community integration, } \\
\text { social contacts or social } \\
\text { interactions and } \\
\text { socialization) }\end{array}$ \\
\hline & $\begin{array}{l}\text { Everyday life } \\
\text { rehabilitation }\end{array}$ & $\begin{array}{l}\text { - Weekly meeting for approximately } 1 \text { to } 2 \mathrm{~h} \\
\text { Set personal goals such as regular walks, } \\
\text { weekly sauna bathing, social interaction } \\
\text { with friends, initiating small talk with } \\
\text { women, eating lunch in a restaurant, } \\
\text { healthy cooking, and taking control of one's } \\
\text { own pocket money }\end{array}$ & $\begin{array}{l}\text { Self-efficacy (self-esteem } \\
\text { or self-confidence) }\end{array}$ \\
\hline $\begin{array}{l}\text { Vocational } \\
\text { rehabilitation }\end{array}$ & $\begin{array}{l}\text { Individual Placement } \\
\text { and Support (IPS) }\end{array}$ & $\begin{array}{ll}\text { - } & \text { Initial vocational assessments } \\
\text { - } & \text { Job searching } \\
\text { - } & \text { Individual job development } \\
\text { - } & \text { Training and regular supervision } \\
\text { - } & \text { Work performance monitoring } \\
\text { - } & \text { Support for employers and continuing } \\
& \text { post-employment support }\end{array}$ & $\begin{array}{c}\text { Returning to open } \\
\text { employment, gaining } \\
\text { competitive employment, } \\
\text { economic empowerment, } \\
\text { gaining financial literacy } \\
\text { skills, financial } \\
\text { independence and } \\
\text { stability }\end{array}$ \\
\hline
\end{tabular}

Conventional vocational rehabilitation (e.g., sheltered employment, supported employment enterprises and social firms)
- $\quad$ Taking of photographs from daily life and constructing text, exhibition and large group discussion

- $\quad$ Exploring, documenting and sharing ideas about recovery

Photovoice

Narrative photovoice and art making
- Weekly class sessions and community outings and exercises on construction of recovery
Empowerment, community integration, hope, progress in recovery
Art making and exhibition
- Weekly art appreciation class took place for several months

- Art appreciation class includes both classroom sessions and community outings
Development of new skills, community integration

\section{Outcome of Recovery Services}

\subsection{Psychiatric Medication and Treatment}

Ten of the included papers highlighted that recovery services have helped to improve the clinical outcomes of adults living with SMI [24,25,33,36,42,44,48,53,62,63]. The services specifically increased access to psychiatric medication and antipsychotic medication adherence, decreased relapse, improved knowledge and illness management, and decreased clinical contact $[24,25,33,36,42,44,53]$. For instance, Lee, Liem [44] reported that recovery services have improved most outcome parameters such as bed days, readmission episodes and missed psychiatric appointments. Conversely, Malinovsky, Lehrer [48] suggested that the number of days spent in the hospital decreased by approximately $40 \%$ after initiation 
of recovery transformation. Furthermore, some studies suggested that the effects of IPS interventions on the time patients spent in competitive employment have had a significant effect on the clinical status, particularly a reduced need for psychiatric inpatient care [33,42]. For instance, Kilian, Lauber [42] indicated that consumers who received an IPS intervention spent fewer days in the hospital.

\subsection{Improve Functionality}

Fourteen of the included papers concluded that recovery services have improved the functioning of adults living with severe mental illness (Table 3). Recovery services improved social and psychological functioning as well as motor and process abilities of adults living with severe mental illness. More specifically, Asher, Hanlon [24] reported in a study that a CBR intervention improved functioning in adults with schizophrenia (baseline median: WHODAS $=57.5$ and IQR 36.7, 65.1; end line median: WHODAS = 18.4 and IQR 2.4, 46.2). Similarly, Zhou, Zhou [63] showed in a study that the intervention (e.g., rehabilitation training programs such as day treatment, medication monitoring, biweekly rehabilitation training) group had a significant improvement in social and psychological functioning.

\subsection{Reduce Symptoms}

Most of the included papers suggested that recovery services have had a significant improvement in anxiety, psychosocial and mental illness symptoms of adults with severe mental illness [25,30,31,36,42,44-46,53,57,63]. For instance, Chang, Chen [30] reported that anxiety symptoms in an experimental group (music-creation program) improved 6.22 points more than in the control group $(p<0.001)$. Similarly, the mean symptoms (Positive and Negative Syndrome Scale PANSS) in a clubhouse group (e.g., open occupation or employment) decreased from 64.5 to 42.7 compared with the control group-from 51.7 to $57.6(p=0.01)$ [57]. More importantly, Lopez-Navarro, Del Canto [46] concluded that incorporating mindfulness interventions into integrated rehabilitation has the potential to reduce negative symptoms.

\subsection{Improvement in Physical Health and Social Behaviour}

Six of the included papers reported that recovery services have improved the physical health and social behaviour of adults with severe mental illness [24,26,36,45,46,48]. In particular, recovery services have shown improvements in physical health, well-being, adaptation, appearance [24,36,45,48], and quality of life (psychological health) [46] as well as reductions in risk-taking behaviour [26]. For instance, Lopez-Navarro, Del Canto [46] recommended that incorporating mindfulness-based interventions into recovery services can improve psychological health-related quality of life. More specifically, the study indicated that mindfulness interventions account for $38 \%$ of the variance in health-related psychological quality of life [46].

\subsection{Self-Efficacy}

Twenty-one of the included papers described the impact of recovery services on the self-efficacy or self-reliance of adults with severe mental illness (see Table 3). The papers highlighted that recovery services improved self-esteem or self-confidence (e.g., fostered feelings of self-worth) $[26,33,35,47,49-51,57,61]$, hope [24,25,30,32], improvements in thoughts, emotions and better understanding of mental illness $[25,26,33]$. More so, recovery services improved self-care or practical skills of daily life (e.g., bathing, washing clothes, as well as undertaking chores related to food preparation and household cleaning) $[26,32]$ as well as personal empowerment [32,49-51].

Three papers recommend that recovery narrative photovoice and art-making services have positively impacted adults with severe mental illness. Such recovery services have improved the lives of consumers in areas through impacts such as on their sense of identity and independence as well as their ability to tolerate uncertainty, take ownership of choices, learn from the past, and maintain vigilance for relapse $[35,49,50]$. In particular, art-making 
interventions provide opportunities for self-exploration and the development of new skills [35]. More importantly, art making and exhibition help people living with severe mental illness clearly express their feelings and communicate emotions and thoughts, which can be difficult to express using words. In addition, recovery narrative photovoice interventions also have a positive impact on outcomes such as autonomy, readiness for change, inspiration, idealism, transformation of self, acceptance of support, awareness of progress, hope, determination, passion, perseverance, introspection, strength, and sense of connectedness $[49,50]$.

Moreover, four papers recommended that vocational rehabilitation interventions impacted positively on sense of identity (e.g., sense of competence through participation in work), reducing boredom/loneliness, staying anchored in reality and creating strong internal motivation for change $[39,43,51,52]$. The feelings and expressions of clients help them to develop a sense of self-determination and personal recovery [52,54].

\subsection{Economic Empowerment}

Nineteen of the included papers reported that recovery services, for instance, vocational participation, have helped to improve the economic empowerment of adults with severe mental illness (see Table 3). More importantly, vocational rehabilitation programs provide livelihood and income-generating activities [24,26,27,43,51].

Most papers reported that adults living with severe mental illness who participated in vocational interventions gained competitive employment $[27,31,36,56,60]$, returned to open employment [28] and received vocational benefits [38,43,56,60]. In particular, adults living with severe mental illness participating in vocational interventions are more likely to receive job-seeking assistance (e.g., searching for jobs, filling application forms and practice for interviews) $[43,47,60]$.

Most of the studies suggested that vocational interventions such as IPS are more effective than the conventional vocational services, particularly in every vocational outcome. IPS clients are more effective at working competitively, returning to open employment (e.g., working for at least one day), and achieving a longer duration of employment (e.g., working for many hours and longer job tenure) and securing greater wages $[28,29,31,42,43,59]$. Catty, Lissouba [29] reported that IPS clients were two-fold (214 days) more likely to work for a longer duration than vocational service clients (108 days). Conversely, 57\% of IPS clients (a sample of 58 consumers with schizophrenia) worked competitively, compared with $29 \%$ of conventional vocational clients. Similarly, $70 \%$ of IPS participants obtained any paid work, compared with $36 \%$ of conventional vocational clients [59]. More importantly, vocational rehabilitation interventions have helped consumers to gain financial literacy skills (e.g., managing finances) [24-26,33,51], achieve financial independence and stability [43] and recover [24,31].

\subsection{Social Inclusion (Community Integration)}

Twenty-seven of the included papers reported that recovery services have increased social inclusion and community acceptance or integration of adults with severe mental illness (see Table 3). Specifically, recovery services achieve increasing social and community participation (e.g., participating in social activities such as churches, coffee ceremonies weddings and funerals), reduce discrimination [24,56], reduce social isolation, create supportive social environments [25], increase social contact or social interaction [26,39,41,43-45,55,56] and increase socialization (e.g., being around and having breaks and playing games) $[41,55,56]$.

Some studies reported that recovery services such as IPS recovery narrative photovoice and art making help adults with severe mental illness to achieve or re-establish valued roles in the community $[33,35,49]$. Whitley, Harris [61] recommended that consumers with severe mental illness use the community as a place of safety and surrogate family as well as for socialization and individual growth. In addition, vocational participation rehabilitation services increase the social contact between adults with SMI and their supervisors and customers or clients, which subsequently reduces the feeling of social isolation $[39,43,51,54,55]$. 
Furthermore, some studies suggested that social environment recovery services, (e.g., clubhouse used as a community) can create an atmosphere of acceptance and inclusion and subsequently support each member's personal recovery journey [52,54]. For instance, adults with severe mental illness in residential programs (clubhouse) showed greater participation in recreational events and informal socialization with peers [62], more social relationships, greater quality of life $[57,63]$ and feeling valued, and greater inclusion and belonging to a group [38]. Further, De Heer-Wunderink, Visser [34] reported that supported independent living programs seemed to positively influence the level of social inclusion for consumers living with severe mental illness in terms of being active and socializing with others.

\subsection{Household Integration}

Three of the included papers reported that recovery services have achieved integration of adults with severe mental illness into their families $[24,25,36]$. Such recovery services have increased greater participation in household tasks and family stability and care [24,25]. For instance, Asher, Hanlon [24] reported that a recovery-oriented rehabilitation service has helped adults with severe mental illness return home to address the basic needs of shelter and food. The services have also equipped family caregivers to develop resilience to accommodate their relatives, including informing them of plans in advance, communicating calmly and avoiding stressors. Consequently, the services have helped to reduce caregiver burden as well as treating adults with severe mental illness with dignity and effective caregiving (e.g., providing food, shelter and shelter) [24].

\subsection{Social Support}

Seven of the included papers highlighted several support services used to implement recovery services for adults with SMI $[31,33,34,36,39,50]$. Some papers highlighted that support services originate from sources such as relatives, friends or peers $[34,39]$ as well as supervisor support and community peers [33,39]. In particular, De Heer-Wunderink, Visser [34] suggested that more than $85 \%$ of clients in recovery-oriented rehabilitation service reported having received support from a partner, their family, or friends. Conversely, Harpaz-Rotem, Rosenheck [36] reported that clients receiving a residential treatment had significantly higher social support on average $(p<0.001)$ after baseline. Supervisors (staff) from recovery services provide practical and emotional support to adults with severe mental illness [39,61]. Whitley, Harris [61] reported that most adults with severe mental illness considered supervisors or staff to be equally important members of their surrogate family.

\section{Discussion}

This review was conducted to synthesize evidence on recovery services used to improve the lives of adults living with severe mental illness. The review findings are discussed according to two emerging themes: (i) mechanisms for implementing recovery services and (ii) outcome of recovery services.

\subsection{Mechanisms for Implementing Recovery Services}

Recovery services are interventions that aim to provide person-centred mental health services, through a whole-system or holistic approach towards the recovery journey of consumers [5]. The review findings identified several recovery services including integrated recovery services, vocational rehabilitation and recovery narrative photovoice and artmaking exhibitions. Integrated recovery services, for instance, are offered through illness management, mindfulness interventions, and task-shifting approaches (e.g., participatorybased training) as well as home visiting, active leisure and music therapy services. Integrated recovery services are mostly incorporated into conventional services and aim to achieve holistic mental health services. The review findings encourage service providers to integrate mindfulness practices, active leisure, music therapy and spiritual healing practices as part of integrated recovery service models $[30,46,56]$. The inclusion of such components 
not only ameliorates the symptoms but is also useful in achieving a sense of agency and autonomy, taking personal responsibility and getting on with life [6]. Conversely, integrated recovery services encourage the use of task shifting and home visiting to promote the recovery journey $[24-26,40,44]$. Home visiting and task shifting can help consumers take up a central role in managing illness (e.g., regaining control) and personal growth as well as establishing a fulfilling and meaningful life [1]. Task shifting and home visiting can help consumers and their families to set personal goals towards their recovery journey. Our review findings encourage service providers to implement integrated recovery service models to improve the lives of consumers.

The evidence suggests that vocational rehabilitation services are also increasingly employed to promote the recovery process of consumers This service is mostly offered through IPS, supported employment enterprises and social firms compared with conventional vocational services (e.g., sheltered employment) [28,29]. Vocational rehabilitation services that are used to support the recovery process of consumers are consistent with previous literature $[10,12,13]$. In particular, IPS interventions have been proven to improve the recovery journey. IPS interventions identified in the current findings are mostly associated with agricultural production, creative projects, services and clients joining the labour market $[28,29,43,57]$. The interventions are usually implemented through initial vocational assessment, job searching, individual job development, monitoring work performance, support for employers and continuing post-employment support for clients. The review findings encourage service providers to implement vocational rehabilitation services (e.g., IPS interventions) that are contextually respected by local service providers and communities. More specifically, researchers are encouraged to use interventional studies to measure the effectiveness of different vocational rehabilitation services that aim to promote the recovery process of consumers.

Recovery narrative photovoice, art-making and exhibition interventions have recently been employed as recovery services to support the recovery process $[32,35,41,49,50]$. Photovoice, art making and exhibition services are implemented through text construction and photographs. This service is also presented through exhibition and large group discussion. This service is particularly employed to achieve recovery, empowerment, community integration, express difficult experiences in non-verbal forms as well as avoid the stigma associated with conventional mental health services. The review findings encourage researchers to use interventional studies to explore the effectiveness of recovery narrative photovoice and art-making exhibition services towards the recovery journey, particularly in multi-cultural settings, where there is increased stigmatization towards mental illness. Such interventional studies could help consumers to develop culturally sensitive recovery goals.

\subsection{Outcome of Recovery Services}

Recovery services necessarily focus on enhancing consumer's capacities for living with, managing, and pursuing a life in the presence of disability, as well as removing barriers around their environment [7]. Consistent with earlier studies, current recovery services are useful in enhancing psychiatric medication and treatment (clinical outcomes) of consumers [3,7]. More specifically, such services increase access to psychiatric medication, antipsychotic medication adherence, decrease relapse, improve knowledge and decrease clinical contact $[24,25,33,36,42,44,53]$. These services are not only about helping consumers to learn how to live a fuller and more satisfying life but also contribute to the reduction in the symptom itself. The review findings encourage service providers to promote holistic care that considers the individual's subjective appraisal of his or her functioning and satisfaction with life [8].

In addition, psychiatric rehabilitation has recently moved beyond the mere control of symptoms and prevention of relapse to incorporate a functional recovery and enhancement of the quality of life of the consumer [1]. The ability of service providers to improve the quality of life of consumers could help to achieve a personal recovery process that is consumer-centred. The current review findings demonstrated that recovery services 
have supported the physical health and social behaviour of consumers. More specifically, such recovery services improve physical health, well-being, adaptation, appearance, and quality of life and reduce risk-taking behaviour [24,26,36,45,46,48]. Improvement in the physical and social behaviour of consumers could also help them to progress in developing selfefficacy, self-confidence, and gaining hope, improvements in self-care or practical skills. The interventions have specifically enhanced the recovery process through readiness for change, autonomy, inspiration, idealism, sense of connectedness and transformation of self.

Recovery services have also improved the economic empowerment of adults living with severe mental illness. The services provide livelihood and income-generating avenues that can facilitate access to competitive employment, returning to open employment and vocational benefits. It is apparent that the participation in income-generating activities improves the financial literacy skills, financial independence and financial stability of adults living with severe mental illness. The improvement in finances through income-generating activities forms a major component of the recovery journey or process. The findings confirm previous literature which suggest that recovery services could empower adults with severe mental illness, through normative life participation such as education, social and political activities [1,2]. The major strength of recovery services mostly relies on its ability to safeguard empowerment in the consumers through everyday living skills, accommodation, social networks, employment and education endeavours [2]. Again, the review findings demonstrated that recovery services aid social inclusion and community acceptance of adults living with severe mental illness. Consistent with previous literature, recoveryoriented rehabilitation promotes the inclusion of adults with severe mental illness through increasing community participation, social contact or social interaction and socialization as well as creating supportive social environments [3]. Such services support consumers to reconnect and re-establish a place in the community, and to explore opportunities that could help them live an independent life. Consequently, the services reduce social isolation, discrimination and stigmatization among consumers [2]. In addition, consumers are also integrated greatly into their individual families and so take an active role in family activities and also maintain family stability.

\section{Limitations}

This review has several limitations that need consideration. The limitations of the integrative review are largely pertinent to the search words, language limitations, and period of included papers. This review was limited to papers published in the English Language from January 2008 to January 2020. In particular, limiting studies to only English Language articles published between January 2008 to January 2020 could miss relevant non-English Language articles as well as those published before 2008. The variation in search terms and keywords regarding recovery services may miss some relevant articles. Additionally, each included study uses different tools for assessing the clinical and personal recovery of the included consumers. This does not allow distinguishing which intervention is most useful. In addition, participants (adults with different mental illnesses) may have different prognoses in different interventions. Given that we did not perform a meta-analysis, there is no objective measure of the effect size of included papers to determine the outcome of recovery. Despite these limitations, this study has some strengths. For example, the combination of clearly articulated search methods, consultation with a research librarian, and reviewing articles with multiple experts as well as the quality assessment tool used to measure the methodological quality helped to address the various limitations.

\section{Conclusions}

The review findings showed that several studies have been undertaken regarding recovery services that can improve the lives of adults living with severe mental illness. Most recovery services are implemented in developed western countries, particularly in the USA and Europe, with relatively few studies piloted in developing countries (for example Africa). The review findings demonstrated that most of the papers used quantita- 
tive data, with few studies employing both qualitative and quantitative data to achieve complementarity or convergence. The evidence showed that most recovery services are delivered through community-based settings. Additionally, studies on recovery services largely address issues on integrated recovery service and vocational rehabilitation, with few studies addressing recovery narrative photovoice and art-making exhibition services. Furthermore, recovery services are reported to be relevance in areas such as medication and treatment adherence, functioning, symptoms, physical health and social behaviour, selfefficacy, economic empowerment, social inclusion or community integration, household integration and access to social support services.

\section{Implications for Mental Health Policy and Practice}

Based on the findings, we recommend that awareness and advocacy for recovery services should be prioritised in national and international policy initiatives. Specifically, consumer associations, self-help groups and family caregivers could be empowered to take the leading advocacy role in recovery services. Additionally, such recovery services should be prioritised by clinicians and allied health professionals to support the recovery goals of consumers. Further research on recovery services should be prioritized in clinical practice and directed towards interventional studies, which can provide sustainable and workable solutions in the recovery journey and outcomes. Given that recovery research mostly employs quantitative and qualitative methods, we recommend that future recovery research should attempt to use mixed methods to achieve complementarity and congruence in both methods.

Supplementary Materials: The following are available online at https:/ / www.mdpi.com/article/ 10.3390/ijerph18168873/s1, Table S1: Data extraction forms, Table S2: Methodological Quality assessment Criteria.

Author Contributions: E.B., A.P.O. and R.M. conceptualized this study; E.B. conducted the data extraction; A.P.O. and R.M. conducted the second review of the extracted data; E.B., working closely with A.P.O. and R.M. performed the analysis and drafted the manuscript; E.B., A.P.O. and R.M. reviewed and made inputs into the intellectual content and agreed on its submission for publication. All authors have read and agreed to the published version of the manuscript.

Funding: This research received no external funding.

Data Availability Statement: All data generated or analysed during this study are included in this article and its Supplementary Information files.

Acknowledgments: The authors wish to thank the University of Newcastle Graduate Research Australia, for the Doctoral Scholarship offered to the lead author.

Conflicts of Interest: The authors declare no conflict of interest.

$\begin{array}{ll}\text { Abbreviations } & \\ & \\ \text { Individual Placement Services } & \text { (IPS); } \\ \text { Joanna Briggs Institute } & (\mathrm{JBI}) ; \\ \text { Mixed-Methods Appraisal Tool } & \text { (MMAT); } \\ \text { Preferred Reporting Items for Systematic Reviews and Meta-Analyses } & \text { (PRISMA); } \\ \text { Severe Mental Illness } & \text { (SMI); } \\ \text { World Health Organization Disability Assessment Scale } & \text { (WHODAS). }\end{array}$

\section{References}

1. Yildiz, M. Recovery as a process in severe mental illnesses. Nöro Psikiyatr. Arş. 2015, 52, 1. [CrossRef]

2. Frost, B.G.; Tirupati, S.; Johnston, S.; Turrell, M.; Lewin, T.J.; Sly, K.A.; Conrad, A.M. An Integrated Recovery-oriented Model (IRM) for mental health services: Evolution and challenges. BMC Psychiatry 2017, 17, 22. [CrossRef] [PubMed]

3. Gopal, S.; Henderson, A.R. Trans-cultural study of recovery from severe enduring mental illness in Chennai, India and Perth, Western Australia. J. Psychosoc. Rehabil. Ment. Health 2015, 2, 51-57. [CrossRef]

4. Slade, M.; Longden, E. Empirical evidence about recovery and mental health. BMC Psychiatry 2015, 15, 285. [CrossRef] 
5. Drake, R.E.; Whitley, R. Recovery and Severe Mental Illness: Description and Analysis. Can. J. Psychiatry Rev. Can. Psychiatr. 2014, 59, 236-242. [CrossRef] [PubMed]

6. Noordsy, D.; Torrey, W.; Mueser, K.; Mead, S.; O'Keefe, C.; Fox, L. Recovery from severe mental illness: An intrapersonal and functional outcome definition. Int. Rev. Psychiatry 2002, 14, 318-326. [CrossRef]

7. Davidson, L.; Roe, D. Recovery from versus recovery in serious mental illness: One strategy for lessening confusion plaguing recovery. J. Ment. Health 2007, 16, 459-470. [CrossRef]

8. Psych, B.; Remington, G.; Lee, J. Personal recovery in serious mental illness: Making sense of the concept. Ann. Acad. Med. Singap. 2017, 46, 29-31.

9. Badu, E.; O'Brien, A.P.; Mitchell, R. The Conceptualization of Mental Health Service Quality Assessment: Consumer Perspective. Adm. Policy Ment. Health Ment. Health Serv. Res. 2019, 26, 790-806. [CrossRef] [PubMed]

10. Gibson, R.W.; D'Amico, M.; Jaffe, L.; Arbesman, M. Occupational Therapy Interventions for Recovery in the Areas of Community Integration and Normative Life Roles for Adults with Serious Mental Illness: A Systematic Review. Am. J. Occup. Ther. 2011, 65, 247-256. [CrossRef] [PubMed]

11. Gyamfi, N.; Badu, E.; Mprah, W.K.; Mensah, I. Recovery services and expectation of consumers and mental health professionals in community-based residential facilities of Ghana. BMC Psychiatry 2020, 20, 355. [CrossRef] [PubMed]

12. Metcalfe, J.D.; E Drake, R.; Bond, G.R. Economic, Labor, and Regulatory Moderators of the Effect of Individual Placement and Support Among People with Severe Mental Illness: A Systematic Review and Meta-analysis. Schizophr. Bull. 2018, 44, 22-31. [CrossRef] [PubMed]

13. Gmitroski, T.; Bradley, C.; Heinemann, L.; Liu, G.; Blanchard, P.; Beck, C.; Mathias, S.; Leon, A.; Barbic, S.P. Barriers and facilitators to employment for young adults with mental illness: A scoping review. BMJ Open 2018, 8, e024487. [CrossRef]

14. Whittemore, R.; Knafl, K. The integrative review: Updated methodology. J. Adv. Nurs. 2005, 52, 546-553. [CrossRef]

15. Hopia, H.; Latvala, E.; Liimatainen, L. Reviewing the methodology of an integrative review. Scand. J. Caring Sci. 2016, 30, 662-669. [CrossRef] [PubMed]

16. National Institute of Mental Health. Mental Illness. 2018. Available online: https://www.nimh.nih.gov/health/statistics/mentalillness.shtml (accessed on 15 December 2020).

17. Pratt, L.A. Characteristics of Adults with Serious Mental Illness in the United States Household Population in 2007. Psychiatr. Serv. 2012, 63, 1042-1046. [CrossRef] [PubMed]

18. Pearson, A.; White, H.; Bath-Hextall, F.; Apostolo, J.; Salmond, S.; Kirkpatrick, P. Methodology for JBI mixed methods systematic reviews. Joanna Briggs Inst. Rev. Man. 2014, 1, 5-34.

19. Moher, D.; Liberati, A.; Tetzlaff, J.; Altman, D.G. On behalf of the PRISMA Group. Preferred Reporting Items for Systematic Reviews and Meta-Analyses: The PRISMA Statement. PLoS Med. 2009, 6, e1000097. [CrossRef]

20. Hong, Q.N.; Fàbregues, S.; Bartlett, G.; Boardman, F.; Cargo, M.; Dagenais, P.; Gagnon, M.-P.; Griffiths, F.; Nicolau, B.; O'Cathain, A.; et al. The Mixed Methods Appraisal Tool (MMAT) version 2018 for information professionals and researchers. Educ. Inf. 2018, 34, 285-291. [CrossRef]

21. The Joanna Briggs Institute (JBI). Critical Appraisal Tools. 2017 [cited 2017 June 19]. Available online: http:/ /joannabriggs.org/ research/critical-appraisal-tools.html (accessed on 19 June 2021).

22. Braun, V.; Clarke, V. Using thematic analysis in psychology. Qual. Res. Psychol. 2006, 3, 77-101. [CrossRef]

23. Fossey, E.; Harvey, C.; McDermott, F.; Davidson, L. Understanding and Evaluating Qualitative Research. Aust. N. Z. J. Psychiatry 2002, 36, 717-732. [CrossRef]

24. Asher, L.; Hanlon, C.; Birhane, R.; Habtamu, A.; Eaton, J.; Weiss, H.A.; Patel, V.; Fekadu, A.; De Silva, M. Community-based rehabilitation intervention for people with schizophrenia in Ethiopia (RISE): A 12 month mixed methods pilot study. BMC Psychiatry 2018, 18, 250. [CrossRef] [PubMed]

25. Brooke-Sumner, C.; Lund, C.; Selohilwe, O.; Petersen, I. Community-based psychosocial rehabilitation for schizophrenia service users in the north west province of South Africa: A formative study. Soc. Work. Ment. Health 2016, 15, 249-283. [CrossRef]

26. Brooke-Sumner, C.; Selohilwe, O.; Mazibuko, M.S.; Petersen, I. Process Evaluation of a Pilot Intervention for Psychosocial Rehabilitation for Service Users with Schizophrenia in North West Province, South Africa. Community Ment. Health J. 2018, 54, 1089-1096. [CrossRef] [PubMed]

27. Browne, D.J.; Waghorn, G. Employment services as an early intervention for young people with mental illness. Early Interv. Psychiatry 2010, 4, 327-335. [CrossRef] [PubMed]

28. Burns, T.; White, S.J.; Catty, J. Individual placement and support in Europe: The EQOLISE trial. Int. Rev. Psychiatry 2018, 20, 498-502. [CrossRef]

29. Catty, J.; Lissouba, P.; White, S.; Becker, T.; Drake, R.E.; Fioritti, A.; Knapp, M.; Lauber, C.; Rössler, W.; Tomov, T.; et al. Predictors of employment for people with severe mental illness: Results of an international six-centre randomised controlled trial. $\mathrm{Br}$. $\mathrm{J}$. Psychiatry 2008, 192, 224-231. [CrossRef]

30. Chang, B.-H.; Chen, B.-W.; Beckstead, J.W.; Yang, C.-Y. Effects of a music-creation programme on the anxiety, self-esteem, and quality of life of people with severe mental illness: A quasi-experimental design. Int. J. Ment. Health Nurs. 2018, 27, 1066-1076. [CrossRef]

31. Chiu, M.Y.L.; Ho, W.W.N.; Lo, W.T.L.; Yiu, M.G.C. Operationalization of the SAMHSA model of recovery: A quality of life perspective. Qual. Life Res. 2009, 19, 1-13. [CrossRef] 
32. Clements, K. Participatory action research and photovoice in a psychiatric nursing/clubhouse collaboration exploring recovery narrative. J. Psychiatr. Ment. Health Nurs. 2012, 19, 785-791. [CrossRef]

33. Crain, M.; Penhale, C.; Newstead, C.; Thomson, L.; Heah, T.; Barclay, K. The contribution of IPS to recovery from serious mental illness: A case study. Work 2009, 33, 459-464. [CrossRef]

34. De Heer-Wunderink, C.; Visser, E.; Sytema, S.; Wiersma, D. Social Inclusion of People With Severe Mental Illness Living in Community Housing Programs. Psychiatr. Serv. 2012, 63, 1102-1107. [CrossRef]

35. Fenner, P.; Ryan, B.; Latai, L.; Percival, S. Art making and the promotion of wellbeing in Samoa-Participants' lived experience of a recovery oriented intervention. Arts Health 2018, 10, 124-137. [CrossRef]

36. Harpaz-Rotem, I.; Rosenheck, R.A.; Desai, R. Residential treatment for homeless female veterans with psychiatric and substance use disorders: Effect on 1-year clinical outcomes. J. Rehabil. Res. Dev. 2011, 48, 891-900. [CrossRef]

37. Hultqvist, J.; Markström, U.; Tjörnstrand, C.; Eklund, M. Quality of life among people with psychiatric disabilities attending community-based day centres or Clubhouses. Scand. J. Caring Sci. 2018, 32, 1418-1427. [CrossRef] [PubMed]

38. Hultqvist, J.; Markström, U.; Tjörnstrand, C.; Eklund, M. Programme characteristics and everyday occupations in day centres and clubhouses in Sweden. Scand. J. Occup. Ther. 2016, 24, 197-207. [CrossRef] [PubMed]

39. Iancu, S.C.; Zweekhorst, M.B.M.; Veltman, D.J.; Van Balkom, A.J.L.M.; Bunders, J.F.G. Mental health recovery on care farms and day centres: A qualitative comparative study of users' perspectives. Disabil. Rehabil. 2013, 36, 573-583. [CrossRef] [PubMed]

40. Iwasaki, Y.; Coyle, C.; Shank, J.; Messina, E.; Porter, H. Leisure-Generated Meanings and Active Living for Persons with Mental Illness. Rehabil. Couns. Bull. 2013, 57, 46-56. [CrossRef]

41. Ketch, R.A.; Rubin, R.T.; Baker, M.R.; Sones, A.C.; Ames, D. Art appreciation for veterans with severe mental illness in a VA Psychosocial Rehabilitation and Recovery Center. Arts Health 2015, 7, 172-181. [CrossRef]

42. Kilian, R.; Lauber, C.; Kalkan, R.; Dorn, W.; Rössler, W.; Wiersma, D.; Van Buschbach, J.T.; Fioritti, A.; Tomov, T.; Catty, J.; et al. The relationships between employment, clinical status, and psychiatric hospitalisation in patients with schizophrenia receiving either IPS or a conventional vocational rehabilitation programme. Soc. Psychiatry Psychiatr. Epidemiol. 2011, 47, 1381-1389. [CrossRef] [PubMed]

43. Koletsi, M.; Niersman, A.; Van Busschbach, J.T.; Catty, J.; Becker, T.; Burns, T.; Fioritti, A.; Kalkan, R.; Lauber, C.; Rössler, W.; et al. Working with mental health problems: Clients' experiences of IPS, vocational rehabilitation and employment. Soc. Psychiatry Psychiatr. Epidemiol. 2009, 44, 961-970. [CrossRef]

44. Lee, C.C.; Liem, S.K.; Leung, J.; Young, V.; Wu, K.; Kenny, K.K.W.; Yuen, S.K.; Lee, W.F.; Leung, T.; Shum, M.; et al. From deinstitutionalization to recovery-oriented assertive community treatment in Hong Kong: What we have achieved. Psychiatry Res. 2015, 228, 243-250. [CrossRef]

45. Lindström, M.; Hariz, G.-M.; Bernspång, B. Dealing with Real-Life Challenges: Outcome of a Home-Based Occupational Therapy Intervention for People with Severe Psychiatric Disability. OTJR Occup. Particip. Health 2011, 32, 5-14. [CrossRef]

46. López-Navarro, E.; Del Canto, C.; Belber, M.; Mayol, A.; Fernández-Alonso, O.; Lluis, J.; Munar, E.; Chadwick, P. Mindfulness improves psychological quality of life in community-based patients with severe mental health problems: A pilot randomized clinical trial. Schizophr. Res. 2015, 168, 530-536. [CrossRef] [PubMed]

47. Luk, A.L. Investigating the long-term effects of a psychiatric rehabilitation programme for persons with serious mental illness in the community: A follow-up study. J. Clin. Nurs. 2011, 20, 2712-2720. [CrossRef]

48. Malinovsky, I.; Lehrer, P.; Silverstein, S.M.; Shankman, S.A.; O’Brien, W.; Samuelson, T.; van Nostrand, G. An empirical evaluation of recovery transformation at a large community psychiatric rehabilitation organization. Psychol. Serv. 2013, 10, 428-441. [CrossRef] [PubMed]

49. Mizock, L.; Russinova, Z.; DeCastro, S. Recovery Narrative Photovoice: Feasibility of a Writing and Photography Intervention for Serious Mental Illnesses. Psychiatr. Rehabil. J. 2015, 38, 279-282. [CrossRef]

50. Mizock, L.; Russinova, Z.; Shani, R. New roads paved on losses: Photovoice perspectives about recovery from mental illness. Qual. Health Res. 2014, 24, 1481-1491. [CrossRef] [PubMed]

51. Panczak, A.; Pietkiewicz, I. Work activity in the process of recovery-An interpretive phenomenological analysis of the experiences of people with a schizophrenia spectrum diagnosis. Psychiatr. Pol. 2016, 50, 805-826. [CrossRef] [PubMed]

52. Raeburn, T.; Schmied, V.; Hungerford, C.; Cleary, M. The use of social environment in a psychosocial clubhouse to facilitate recovery-oriented practice. BJPsych Open 2016, 2, 173-178. [CrossRef] [PubMed]

53. Salyers, M.P.; McGuire, A.B.; Kukla, M.; Fukui, S.; Lysaker, P.; Mueser, K.T. A Randomized Controlled Trial of Illness Management and Recovery with an Active Control Group. Psychiatr. Serv. 2014, 65, 1005-1011. [CrossRef]

54. Svanberg, J.; Gumley, A.; Wilson, A. How do social firms contribute to recovery from mental illness? A qualitative study. Clin. Psychol. Psychother. 2010, 17, 482-496. [CrossRef]

55. Swildens, W.; van Busschbach, J.T.; Michon, H.; Kroon, H.; Koeter, M.W.J.; Wiersma, D.; van Os, J. Effectively Working on Rehabilitation Goals: 24-Month Outcome of a Randomized Controlled Trial of the Boston Psychiatric Rehabilitation Approach. Can. J. Psychiatry 2011, 56, 751-760. [CrossRef]

56. Tjörnstrand, C.; Bejerholm, U.; Eklund, M. Participation in day centres for people with psychiatric disabilities: Characteristics of occupations. Scand. J. Occup. Ther. 2011, 18, 243-253. [CrossRef]

57. Tsang, A.W.K.; Ng, R.M.K.; Yip, K.C. A six-month prospective case-controlled study of the effects of the clubhouse rehabilitation model on Chinese patients with chronic schizophrenia. East Asian Arch. Psychiatry 2010, 20, 23. 
58. Tondora, J.; O'Connell, M.; Miller, R.; Dinzeo, T.; Bellamy, C.; Andres-Hyman, R.; Davidson, L. A clinical trial of peer-based culturally responsive person-centered care for psychosis for African Americans and Latinos. Clin. Trials 2010, 7, 368-379. [CrossRef] [PubMed]

59. Twamley, E.W.; Vella, L.; Burton, C.Z.; Becker, D.R.; Bell, M.D.; Jeste, D.V. The efficacy of supported employment for middle-aged and older people with schizophrenia. Schizophr. Res. 2012, 135, 100-104. [CrossRef]

60. Waghorn, G.; Dias, S.; Gladman, B.; Harris, M. Measuring what matters: Effectiveness of implementing evidence-based supported employment for adults with severe mental illness. Int. J. Ther. Rehabil. 2015, 22, 411-420. [CrossRef]

61. Whitley, R.; Harris, M.; Fallot, R.D.; Berley, R.W. The active ingredients of intentional recovery communities: Focus group evaluation. J. Ment. Health 2008, 17, 173-182. [CrossRef]

62. Zemore, S.E.; Kaskutas, L.A. Services received and treatment outcomes in day-hospital and residential programs. J. Subst. Abus. Treat. 2008, 35, 232-244. [CrossRef] [PubMed]

63. Zhou, Y.; Zhou, R.; Li, W.; Lin, Y.; Yao, J.; Chen, J.; Shen, T. Controlled trial of the effectiveness of community rehabilitation for patients with schizophrenia in Shanghai, China. Shanghai Arch. Psychiatry 2015, 27, 167-174. [CrossRef] [PubMed] 